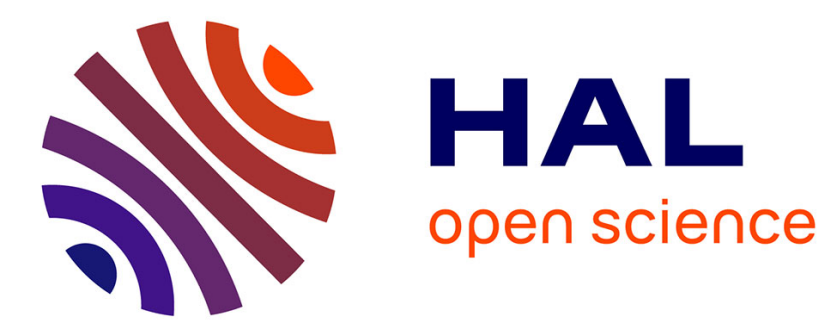

\title{
The recursive variational Gaussian approximation (R-VGA)
}

\author{
Marc Lambert, Silvere Bonnabel, Francis Bach
}

\section{To cite this version:}

Marc Lambert, Silvere Bonnabel, Francis Bach. The recursive variational Gaussian approximation (R-VGA). Statistics and Computing, In press, 10.1007/s11222-021-10068-w . hal-03086627v2

\section{HAL Id: hal-03086627 \\ https://hal.inria.fr/hal-03086627v2}

Submitted on 7 Dec 2021

HAL is a multi-disciplinary open access archive for the deposit and dissemination of scientific research documents, whether they are published or not. The documents may come from teaching and research institutions in France or abroad, or from public or private research centers.
L'archive ouverte pluridisciplinaire HAL, est destinée au dépôt et à la diffusion de documents scientifiques de niveau recherche, publiés ou non, émanant des établissements d'enseignement et de recherche français ou étrangers, des laboratoires publics ou privés. 


\title{
The recursive variational Gaussian approximation (R-VGA)
}

\author{
Marc Lambert \\ DGA/CATOD, Centre d'Analyse Technico-Opérationelle de Défense \\ \& INRIA \\ marc-h. lambert@intradef .gouv.fr \\ Silvère Bonnabel \\ ISEA, Université de la Nouvelle-Calédonie \\ \& MINES ParisTech, PSL University, Center for robotics \\ silvere.bonnabel@mines-paristech.fr \\ Francis Bach \\ INRIA - Ecole Normale Supérieure - PSL Research university \\ francis.bach@inria.fr
}

\begin{abstract}
We consider the problem of computing a Gaussian approximation to the posterior distribution of a parameter given $N$ observations and a Gaussian prior. Owing to the need of processing large sample sizes $N$, a variety of approximate tractable methods revolving around online learning have flourished over the past decades. In the present work, we propose to use variational inference (VI) to compute a Gaussian approximation to the posterior through a single pass over the data. Our algorithm is a recursive version of variational Gaussian approximation we have called recursive variational Gaussian approximation (R-VGA). We start from the prior, and for each observation we compute the nearest Gaussian approximation in the sense of Kullback-Leibler divergence to the posterior given this observation. In turn, this approximation is considered as the new prior when incorporating the next observation. This recursive version based on a sequence of optimal Gaussian approximations leads to a novel implicit update scheme which resembles the online Newton algorithm, and which is shown to boil down to the Kalman filter for Bayesian linear regression. In the context of Bayesian logistic regression the implicit scheme may be solved, and the algorithm is shown to perform better than the extended Kalman filter, while being less computationally demanding than its sampling counterparts.
\end{abstract}

\section{Introduction}

Recent applications of probabilistic modeling and Bayesian inference involve large numbers of observations. In this setting, recursive algorithms that update the posterior distribution after each observation are desirable. In the present paper we seek a Gaussian approximation $q(\theta)=\mathscr{N}(\theta \mid \mu, P)$ to the posterior

$$
p\left(\theta \mid y_{1}, \ldots, y_{N}\right)=\frac{p_{0}(\theta) p\left(y_{1}, \ldots, y_{N} \mid \theta\right)}{p\left(y_{1}, \ldots, y_{N}\right)}=\frac{p_{0}(\theta) p\left(y_{1}, \ldots, y_{N} \mid \theta\right)}{\int p_{0}(\theta) p\left(y_{1}, \ldots, y_{N} \mid \theta\right) d \theta}
$$

given $N$ conditionally independent observations $y_{1}, \ldots, y_{N}$ and a Gaussian prior $p_{0}$. Various methods exist for Gaussian approximation, for instance the Laplace approximation computes the Hessian of the log-posterior at the maximum a posteriori (MAP) to fit the covariance term, whereas moment matching methods estimate the mean and covariance matrix through direct integration [Bishop, 2006]. Besides, variational inference (VI) has become widely used to approximate posteriors. Following this framework, we seek to minimize the 
KL divergence between a Gaussian distribution $q(\theta)=\mathscr{N}(\theta \mid \mu, P)$ and the true posterior. The parameters $\mu$ (mean vector) and $P$ (covariance matrix) have to be estimated in an online fashion. Since the observations are independent conditionally on $\theta$ we have:

$$
\begin{aligned}
& \min _{\mu, P} \quad K L\left(q(\theta) \| p\left(\theta \mid y_{1}, \ldots, y_{N}\right)\right):=\min _{\mu, P} \quad \mathbf{E}_{q}\left[\log \frac{q(\theta)}{p\left(\theta \mid y_{1}, \ldots, y_{N}\right)}\right] \\
= & \min _{\mu, P} \quad \mathbf{E}_{q}\left[\log q(\theta)-\log p_{0}(\theta)\right]-\sum_{i=1}^{N} \mathbf{E}_{q}\left[\log p\left(y_{i} \mid \theta\right)\right]+\log p\left(y_{1}, \ldots, y_{N}\right) .
\end{aligned}
$$

The black box variational inference algorithm [Ranganath et al., 2014] attacks this VI optimization problem using Monte-Carlo samples $\theta_{t} \sim q$, combined with a Robbins-Monro stochastic gradient algorithm [Robbins and Monro, 1951]. The natural gradient algorithm has also been used to improve the rate of convergence using second order information [Lin et al., 2019a]. These algorithms need to parse the entire dataset at each step and online versions have been used on large size datasets to prevent memory overflow [Titsias and Lázaro-Gredilla, 2014].

In this paper we consider recursive variational Bayes inference [Broderick et al., 2013] with a Gaussian target distribution. We consider a variational Gaussian approximation with full covariance matrix rather than a mean field approximation [Waterhouse et al., 1996]. Based on the fixed point equation induced by this recursive variational Gaussian approximation scheme, we derive a novel online algorithm we have called RVGA. The R-VGA scheme consists in letting $q_{0}=p_{0}$ and then computing a Gaussian approximation $q_{t}(\theta)=$ $\mathscr{N}\left(\theta_{t} \mid \mu_{t}, P_{t}\right)$ at each step $1 \leq t \leq N$ in the following recursive way. At step $t$, when observation $y_{t}$ comes in, our approximation $q_{t}(\theta)$ exactly minimizes the Kullback-Leibler (KL) divergence to $\propto p\left(y_{t} \mid \theta\right) q_{t-1}(\theta)$, that is, the partial posterior based on the previous approximation $q_{t-1}$ and latest observation $y_{t}$. After a single pass over the data, a Gaussian approximation $q(\theta)=q_{N}(\theta)$ to the full posterior based on a series of optimal approximations is thus obtained. Optimality comes at a price, though, as at each step fixed point equations need to be solved. These fixed point equations form a novel implicit update scheme which resembles the online Newton algorithm, except that it involves expectations over $q_{t}$ instead of $q_{t-1}$. Note that, although they do not yield a readily implementable closed form solution to the optimization problem, implicit schemes have recently gained interest owing to their inherent stability, see for instance Jézéquel et al. [2020] which considers an implicit scheme for online Newton descent [Hazan et al., 2007] applied to logistic regression.

The proposed R-VGA proves optimal when applied to Bayesian linear regression, and is then shown to boil down to the Kalman filter. When the observations belong to an exponential family and the model is linearized, we show also the R-VGA is algebraically equivalent to the extended Kalman filter and to the online natural gradient, extending connections made by earlier work of Ollivier [2018] and Emtiyaz Khan et al. [2017]. In the present paper, the R-VGA is also applied to Bayesian logistic regression. We show the implicit update scheme may then be solved, and the R-VGA's numerical complexity is identical to the extended Kalman filter's, that is, $O\left(d^{2} N\right)$ where $d$ is the dimension of parameter $\theta$ and $N$ is the number of observations. Numerical experiments based on synthetic data illustrate that the R-VGA outperforms the extended Kalman filter (EKF) as well as other variants having similar computational cost. In some challenging cases it even beats the batch Laplace approximation in terms of divergence to the true posterior.

The paper is organized as follows. In Section 3, we introduce the recursive variational Gaussian approximation (R-VGA) scheme and derive an averaged version of the online Newton algorithm. We also show this second order algorithm can be reformulated in an Hessian free and derivative-free versions. In Section 4 we show that in the case where observations are Gaussian and linearly related to the hidden variable $\theta, \mathrm{R}-\mathrm{VGA}$ is algebraically equivalent to the linear Kalman filter and the online Newton algorithm. We extend these results to the context of exponential family distributions with linearized models, and show the approximated explicit version of R-VGA is then equivalent to the extended Kalman filter (EKF) and to the online natural gradient. In Section 5, R-VGA is applied to the logistic regression problem. For benchmark 
purposes we also derive the quadratic Kalman filter as an alternative variant based on quadratic variational approximations, which is close to the one proposed in Sykacek and Roberts [2003], and recall the EKF equations when applied to logistic regression. Finally numerical experiments allow for algorithm comparisons in Section 6.

\section{Related work}

Classical variational Bayesian methods [Waterhouse et al., 1996] use a mean-field approximation of the posterior to compute the KL-divergence with an iterative scheme. Stochastic gradient descent has been used to solve this problem when the number of observations is large. The stochastic variational inference algorithm (SVI) has first been proposed in Wainwright and Jordan [2008] and variants have been used in Titsias and Lázaro-Gredilla [2014] and McInerney et al. [2015]. Rather than using a stochastic algorithm, a recursive version of variational inference has been proposed in Broderick et al. [2013]. This recursive variational principle has been applied for mean field approximation of Dirichlet distributions in the context of large scale machine learning streaming in Broderick et al. [2013]. A recursive mean field approach was also used for Kalman filtering through a particle filter in Smidl and Quinn [2008]. Our approach uses the same recursive variational inference principle but is based on a variational Gaussian approximation and not a variational mean-field approximation. Our target covariance matrix does not have to be sparse and can model the correlation between all variables, contrary to the mean field approach which neglects some correlations.

Closer to our approach, in Sato [2001] a recursive variational inference scheme has been proposed for exponential family model with latent variables but the observations are assumed conjugate to the target to obtain a closed form solution. A variational Gaussian approximation has recently been considered in Khan and Lin [2017] and Hu et al. [2018] where mirror descent or natural gradients are used to compute a minimizer of the KL divergence. They have shown that applying the natural gradient to the KL divergence leads to (explicit) fixed point updates which involve averaging of the gradients. In the present work, we derive implicit updates directly from the critical points of the KL divergence, and we do not apply explicitly a descent approach to compute a minimizer of the KL divergence. Our approach leads to implicit updates, not explicit ones, hence relaxing the need to tune a step size parameter, provided the implicit scheme may be solved. In Sykacek and Roberts [2003], a Kalman filter is used for classification using the variational lower bound proposed in Jaakkola and Jordan [1997] to propagate a Gaussian state recursively. This filter is close to the one we have devised and compared our algorithm to, and called the quadratic Kalman filter (QKF) in Subsection 5.4.3. Experiments (see Section 6) demonstrate that the QKF is less stable than the proposed R-VGA approach.

Finally, the variational Gaussian inference framework has been extended to more complex models such as mixture of Gaussians Lin et al. [2019a] or hierarchical models Daudel et al. [2021] in stochastic versions. Variational Gaussian updates have been used as a building block in these more complex updates in Lin et al. [2019a]. 


\section{The recursive variational Gaussian approximation (R-VGA)}

The recursive variational approximation is detailed in Algorithm 1.

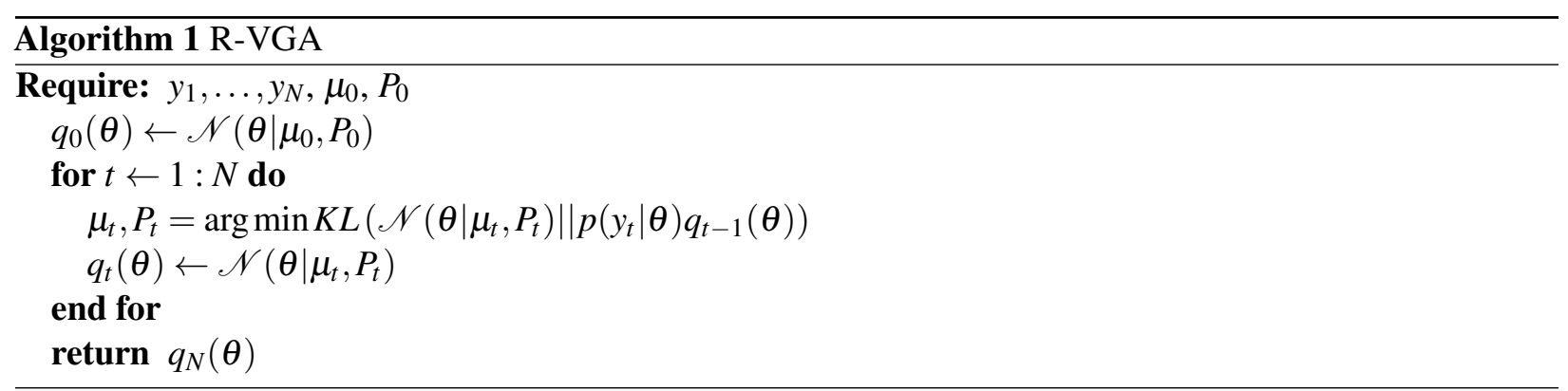

Let $Y_{t}$ denote the observations up to step $t$, that is, $Y_{t}=\left(y_{1}, \ldots, y_{t}\right)$, and $q_{t}$ denote a Gaussian approximation for the distribution of $\theta$ at step $t$, that is, $q_{t}(\theta)=\mathscr{N}\left(\theta \mid \mu_{t}, P_{t}\right)$. The algorithm is based on the recursive update that consists in approximating at each step the distribution $p\left(\theta \mid Y_{t}\right)$ through the past Gaussian approximation $q_{t-1}(\theta)$. More precisely the recursive updates write:

$$
\begin{aligned}
& K L\left(q_{t}(\theta) \| p\left(\theta \mid Y_{t}\right)\right):=\int q_{t}(\theta) \log \frac{q_{t}(\theta)}{p\left(\theta \mid Y_{t}\right)} d \theta \\
& =\int q_{t}(\theta) \log \frac{q_{t}(\theta) p\left(y_{t} \mid Y_{t-1}\right)}{p\left(y_{t} \mid \theta, Y_{t-1}\right) p\left(\theta \mid Y_{t-1}\right)} d \theta \text { (from Bayes' theorem on }\left(\theta, y_{t}\right) \text { ) } \\
& =\int q_{t}(\theta) \log \frac{q_{t}(\theta) p\left(y_{t} \mid Y_{t-1}\right)}{p\left(y_{t} \mid \theta\right) p\left(\theta \mid Y_{t-1}\right)} d \theta \text { (since } y_{t} \text { is conditionally independent of } Y_{t-1} \text { given } \theta \text { ) } \\
& \left.=\int q_{t}(\theta) \log \frac{q_{t}(\theta) p\left(Y_{t}\right)}{p\left(y_{t} \mid \theta\right) p\left(\theta \mid Y_{t-1}\right) p\left(Y_{t-1}\right)} d \theta \text { (multiplying by } \frac{p\left(Y_{t-1}\right)}{p\left(Y_{t-1}\right)}\right) \\
& \approx \int q_{t}(\theta) \log q_{t}(\theta) d \theta-\int q_{t}(\theta) \log p\left(y_{t} \mid \theta\right) d \theta-\int q_{t}(\theta) \log q_{t-1}(\theta) d \theta+\log p\left(Y_{t}\right)-\log p\left(Y_{t-1}\right) .
\end{aligned}
$$

The latter approximation amounts to substituting $p\left(\theta \mid Y_{t-1}\right)$ with the latest computed distribution $q_{t-1}(\theta)$. If the target distribution $q$ is conjugate to the distribution $p$ of the observations, this recursive scheme gives exactly the same result as the batch scheme. In particular, for Gaussian observations through a linear model we will show we obtain the equation of the Kalman filter, which yields the true posterior. In the non linear or non Gaussian case, a cumulative approximation error is then inevitably made.

Based on this approximation, our goal is to compute at each step $t$ the parameters $\mu_{t}$ and $P_{t}$ of the variational distribution $q_{t}$ which minimize the latter expression, that is,

$$
\underset{\mu_{t}, P_{t}}{\operatorname{argmin}} \mathbf{E}_{q_{t}}\left[\log q_{t}(\theta)-\log q_{t-1}(\theta)-\log p\left(y_{t} \mid \theta\right)\right] .
$$

We may compute the equations the minimizers must satisfy at each step $t$. In the next section we show these equations can be written in a form that resembles the online Newton algorithm. Unlike the classical online Newton algorithm which uses a second order Taylor approximation as in the Laplace method, our algorithm is based on a variational approach which involves expectations.

\subsection{The R-VGA as an averaged online Newton algorithm}

Theorem 1 shows the optimal solution at iteration $t$ of the R-VGA can be rewritten as an optimization step of an averaged version of the online Newton descent algorithm. 
Theorem 1. Suppose $\log p(y \mid \theta)$ is absolutely continuous with respect to $\theta$ and the observations $y_{1}, \ldots, y_{N}$ are independent conditionally on $\theta$. Given a Gaussian prior distribution $q_{0}$, a sequence of Gaussian distributions $q_{1}, \ldots, q_{t}$ being solutions to the recursive variational Gaussian approximation $(R-V G A)$ scheme of Algorithm 1 necessarily satisfy the following fixed point equations:

$$
\begin{gathered}
\mu_{t}=\mu_{t-1}+P_{t-1} \nabla_{\mu_{t}} \mathbf{E}_{q_{t}}\left[\log p\left(y_{t} \mid \theta\right)\right] \\
P_{t}^{-1}=P_{t-1}^{-1}-2 \nabla_{P_{t}} \mathbf{E}_{q_{t}}\left[\log p\left(y_{t} \mid \theta\right)\right] .
\end{gathered}
$$

Those fixed point equations are equivalent to a second order form which may be viewed as an averaged version of the online Newton algorithm ("order 2 form"):

$$
\begin{aligned}
& \mu_{t}=\mu_{t-1}+P_{t-1} \mathbf{E}_{q_{t}}\left[\nabla_{\theta} \log p\left(y_{t} \mid \theta\right)\right] \\
& P_{t}^{-1}=P_{t-1}^{-1}-\mathbf{E}_{q_{t}}\left[\nabla_{\theta}^{2} \log p\left(y_{t} \mid \theta\right)\right] .
\end{aligned}
$$

Proof. We seek to minimize the quantity:

$$
\min _{\mu_{t}, P_{t}} \mathbf{E}_{q_{t}}\left[\log q_{t}(\theta)-\log q_{t-1}(\theta)-\log p\left(y_{t} \mid \theta\right)\right]
$$

The critical point with respect to $\mu_{t}$ yields:

$$
\begin{aligned}
& \nabla_{\mu_{t}} \mathbf{E}_{q_{t}}\left[\log q_{t}(\theta)-\log q_{t-1}(\theta)-\log p\left(y_{t} \mid \theta\right)\right] \\
& =\nabla_{\mu_{t}}\left(\frac{1}{2} \mu_{t}^{T} P_{t-1}^{-1} \mu_{t}-\mu_{t-1}^{T} P_{t-1}^{-1} \mu_{t}\right)-\nabla_{\mu_{t}} \mathbf{E}_{q_{t}}\left[\log p\left(y_{t} \mid \theta\right)\right] \\
& =P_{t-1}^{-1} \mu_{t}-P_{t-1}^{-1} \mu_{t-1}-\nabla_{\mu_{t}} \mathbf{E}_{q_{t}}\left[\log p\left(y_{t} \mid \theta\right)\right]=0 \\
& \Longleftrightarrow \mu_{t}=\mu_{t-1}+P_{t-1} \nabla_{\mu_{t}} \mathbf{E}_{q_{t}}\left[\log p\left(y_{t} \mid \theta\right)\right] .
\end{aligned}
$$

The critical point with respect to $P_{t}$ yields:

$$
\begin{aligned}
& \nabla_{P_{t}} \mathbf{E}_{q_{t}}\left[\log q_{t}(\boldsymbol{\theta})-\log q_{t-1}(\boldsymbol{\theta})-\log p\left(y_{t} \mid \boldsymbol{\theta}\right)\right] \\
& =\nabla_{P_{t}}\left(-\frac{1}{2} \log \left|P_{t}\right|+\frac{1}{2} \operatorname{Tr}\left(P_{t} P_{t-1}^{-1}\right)\right)-\nabla_{P_{t}} \mathbf{E}_{q_{t}}\left[\log p\left(y_{t} \mid \boldsymbol{\theta}\right)\right] \\
& =-\frac{1}{2} P_{t}^{-1}+\frac{1}{2} P_{t-1}^{-1}-\nabla_{P_{t}} \mathbf{E}_{q_{t}}\left[\log p\left(y_{t} \mid \boldsymbol{\theta}\right)\right]=0 \\
& \Longleftrightarrow P_{t}^{-1}=P_{t-1}^{-1}-2 \nabla_{P_{t}} \mathbf{E}_{q_{t}}\left[\log p\left(y_{t} \mid \theta\right)\right] .
\end{aligned}
$$

We have thus recovered equations (1) and (2). We operate now a change of variable: the derivative with respect to $\mu$ and $P$ can be transformed into derivatives with respect to $\theta$ using the symmetry properties of the Gaussian distribution:

$$
\begin{aligned}
& \nabla_{\mu} \mathscr{N}(\theta \mid \mu, P)=-\nabla_{\theta} \mathscr{N}(\theta \mid \mu, P) \\
& \nabla_{P} \mathscr{N}(\theta \mid \mu, P)=\frac{1}{2} \nabla_{\theta}^{2} \mathscr{N}(\theta \mid \mu, P) .
\end{aligned}
$$

Since $\log p(y \mid \theta)$ is absolutely continuous with respect to $\theta$, we can interchange differentiation and integration and use the formula of integration by parts, known as the Bonnet \& Price formulas [Lin et al., 2019b], to 
recover equations (3) and (4):

$$
\begin{aligned}
\nabla_{\mu} \mathbf{E}_{q}[\log p(y \mid \theta)] & =\int \log p(y \mid \theta) \nabla_{\mu} \mathscr{N}(\theta \mid \mu, P) d \theta \\
& =-\int \log p(y \mid \theta) \nabla_{\theta} \mathscr{N}(\theta \mid \mu, P) d \theta \\
& =\int \nabla_{\theta} \log p(y \mid \theta) \mathscr{N}(\theta \mid \mu, P) d \theta=\mathbf{E}_{q}\left[\nabla_{\theta} \log p(y \mid \theta)\right] \\
\nabla_{P} \mathbf{E}_{q}[\log p(y \mid \theta)] & =\int \log p(y \mid \theta) \nabla_{P} \mathscr{N}(\theta \mid \mu, P) d \theta \\
& =\frac{1}{2} \int \log p(y \mid \theta) \nabla_{\theta}^{2} \mathscr{N}(\theta \mid \mu, P) d \theta \\
& =-\frac{1}{2} \int \nabla_{\theta} \log p(y \mid \theta) \nabla_{\theta} \mathscr{N}(\theta \mid \mu, P)^{T} d \theta=\frac{1}{2} \mathbf{E}_{q}\left[\nabla_{\theta}^{2} \log p(y \mid \theta)\right],
\end{aligned}
$$

which completes the proof.

Equations (3) and (4) resemble the online Newton algorithm but where the iterates $\theta$ are averaged through expectations. Gradient averaging (over time) has attracted a lot of attention in optimization, see, e.g., Schmidt et al. [2017]. However in our equations, the averaging consists of an expectation over the parameters we are currently estimating and leads to an implicit scheme. We will study this second order implicit scheme in the remainder of this paper and show it can be made explicit in the linear regression case, and can be solved for the logistic regression problem. Before that, the next corollary shows we can derive lower order schemes which are algebraically equivalent to the second order scheme.

Corollary 1.1. The R-VGA updates, in particular the averaged version of the online Newton, can be rewritten in a Hessian-free version ("order 1 form"):

$$
\begin{aligned}
& \mu_{t}=\mu_{t-1}+P_{t-1} \mathbf{E}_{q_{t}}\left[\nabla_{\theta} \log p\left(y_{t} \mid \theta\right)\right] \\
& P_{t}^{-1}=P_{t-1}^{-1}-P_{t}^{-1} \mathbf{E}_{q_{t}}\left[\left(\theta-\mu_{t}\right) \nabla_{\theta} \log p\left(y_{t} \mid \theta\right)^{T}\right],
\end{aligned}
$$

and in a derivative-free version ("order 0 form"):

$$
\begin{aligned}
& \mu_{t}=\mu_{t-1}+P_{t-1} P_{t}^{-1} \mathbf{E}_{q_{t}}\left[\left(\theta-\mu_{t}\right) \log p\left(y_{t} \mid \theta\right)\right] \\
& P_{t}^{-1}=P_{t-1}^{-1}-P_{t}^{-1} \mathbf{E}_{q_{t}}\left[\left(\theta-\mu_{t}\right)\left(\theta-\mu_{t}\right)^{T} \log p\left(y_{t} \mid \theta\right)\right] P_{t}^{-1}+P_{t}^{-1} \mathbf{E}_{q_{t}}\left[\log p\left(y_{t} \mid \theta\right)\right]
\end{aligned}
$$

Proof. The proof is a direct consequence of Theorem 1. Rather than using integration by parts, we use (10) and (13):

$$
\begin{aligned}
\int \log p(y \mid \theta) \nabla_{\mu} \mathscr{N}(\theta \mid \mu, P) d \theta & =\int \log p(y \mid \theta) P^{-1}(\theta-\mu) \mathscr{N}(\theta \mid \mu, P) d \theta \\
& =P^{-1} \mathbf{E}_{q}[(\theta-\mu) \log p(y \mid \theta)] \\
\int \log p(y \mid \theta) \nabla_{P} \mathscr{N}(\theta \mid \mu, P) d \theta & =\int \log p(y \mid \theta)\left(\frac{1}{2} P^{-1}(\theta-\mu)(\theta-\mu)^{T} P^{-1}-\frac{1}{2} P^{-1}\right) \mathscr{N}(\theta \mid \mu, P) d \theta \\
& =\frac{1}{2} P^{-1} \mathbf{E}_{q}\left[(\theta-\mu)(\theta-\mu)^{T} \log p(y \mid \theta)\right] P^{-1}-\frac{1}{2} P^{-1} \mathbf{E}_{q_{t}}[\log p(y \mid \theta)] .
\end{aligned}
$$

Plugging this relation into the R-VGA equations (1) and (2), we recover the new derivative-free update equations of the theorem. The Hessian-free version is a variant where we use only one integration by parts in 
(13):

$$
\begin{aligned}
\int \log p(y \mid \theta) \nabla_{P} \mathscr{N}(\theta \mid \mu, P) d \theta & =-\frac{1}{2} \int \log p(y \mid \theta) \nabla_{\theta} \nabla_{\mu} \mathscr{N}(\theta \mid \mu, P)^{T} d \theta \\
& =\frac{1}{2} \int \nabla_{\theta} \log p(y \mid \theta) \nabla_{\mu} \mathscr{N}(\theta \mid \mu, P)^{T} d \theta \\
& =\frac{1}{2} P^{-1} \mathbf{E}_{q}\left[(\theta-\mu) \nabla_{\theta} \log p(y \mid \theta)^{T}\right],
\end{aligned}
$$

which completes the proof.

These lower order versions open up the possibility of handling complex distributions $\log p(y \mid \theta)$ for which the Hessian or the gradient are difficult to compute or are not well-defined. The Hessian-free version provides a way to compute online the Hessian using only the gradient information. Online versions of Gauss-Newton methods approximate also the Hessian with a gradient, but in Equation (17) the iteration is exact, not approximate, while the scheme is implicit. Derivative-free optimization has been developed for stochastic optimization of a non-smooth function [Nesterov and Spokoiny, 2017]. If the function is smoothed with a Gaussian, integration by parts can be used to eliminate the gradient term. Stochastic derivative-free optimization is known to be much slower than the stochastic gradient counterpart because the smoothing process introduces a bias. Our derivative-free version in Equation (18) is different since it introduces an adaptive step through the equations (19). However these equations are both implicit and not directly implementable.

Theorem 1 can be generalized to any target distribution $q$ that belongs to an exponential family. The R-VGA updates correspond then to an implicit gradient descent on the natural parameters $\eta$ of the exponential family weighted by the inverse of the Hessian of the $\log$ partition function $F$ :

$$
\eta_{t}=\eta_{t-1}+\left(\nabla^{2} F\left(\eta_{t}\right)\right)^{-1} \nabla_{\eta_{t}} \mathbf{E}_{q_{\eta_{t}}}\left[\log p\left(y_{t} \mid \boldsymbol{\theta}\right)\right] .
$$

The interested reader is referred to Appendix 9.1 for more details.

All the equations we have derived until now do not provide explicit iterations. We will discuss now how to construct numerical schemes to implement them.

\subsection{Discussion and closed-form approximations}

We see the implementation of the main R-VGA fixed-point update equations (1)-(2) or any of its variants poses two main difficulties. First, the updates are implicit, i.e., the right-hand side depends on the parameters that one is seeking. Second, they require to compute an expectation over the distribution $q_{t}$. Let us start with the first issue, and devise an approximate explicit scheme.

Explicit approximation: A simple way to approximate the second order implicit scheme (3)-(4):

\section{Implicit scheme (exact R-VGA)}

$$
\begin{aligned}
& \mu_{t}=\mu_{t-1}+P_{t-1} \mathbf{E}_{q_{t}}\left[\nabla_{\theta} \log p\left(y_{t} \mid \theta\right)\right], \\
& P_{t}^{-1}=P_{t-1}^{-1}-\mathbf{E}_{q_{t}}\left[\nabla_{\theta}^{2} \log p\left(y_{t} \mid \theta\right)\right],
\end{aligned}
$$

is to consider $q_{t}$ to be close enough to $q_{t-1}$ and to replace the expectation under $q_{t}$ with the expectation under $q_{t-1}$, yielding the following

\section{Explicit scheme (approximated R-VGA)}

$$
\begin{aligned}
& \mu_{t}=\mu_{t-1}+P_{t} \mathbf{E}_{q_{t-1}}\left[\nabla_{\theta} \log p\left(y_{t} \mid \theta\right)\right], \\
& P_{t}^{-1}=P_{t-1}^{-1}-\mathbf{E}_{q_{t-1}}\left[\nabla_{\theta}^{2} \log p\left(y_{t} \mid \theta\right)\right] .
\end{aligned}
$$


Note we have also changed $P_{t-1}$ into $P_{t}$ in the right hand side of (28), since this makes the above scheme optimal in the linear Gaussian case, see Section 4.1. Generally speaking, the implicit scheme is preferable since it is optimal. However, the explicit scheme may be a viable alternative if the implicit scheme cannot be solved. It comes at a price, but the intuition - confirmed by the experiments - is that if the parameters updated at step $t$ are not far from the previous updates at step $t-1$, the explicit scheme may correctly approach the implicit one since we replace some quantities with quantities that are close by.

Computation of the expectations: Even when using the explicit scheme, one needs to compute expectations under a variational distribution $q$ which is the second issue we have raised. Most often they are analytically intractable, and various approaches have been advocated in the literature. A first approach is to use a Monte Carlo approximation. According to Gal [2016], in the particular case where $\theta$ is a scalar, the Monte Carlo estimator based on $\frac{1}{2} \mathbf{E}_{q}\left[\nabla^{2} f(\theta)\right]$ has lower variance than the one based on $\frac{1}{2} \mathbf{E}_{q}\left[P^{-1}(\theta-\mu) \nabla_{\theta} f(\theta)^{T}\right]$, indicating the second order scheme shall be then preferred. A second approach to the computation of expectations is to use quadrature rules, that compute an approximation to the integral based on a finite number of so-called "sigma points". Such quadrature integrals have recently been advocated by Barfoot et al. [2020] where a derivative-free form was also used in the context of batch variational inference.

In this paper we apply the R-VGA to both Bayesian linear and logistic regression. For logistic regression, we show we can circumvent both issues that have been raised, as solving the implicit scheme is amenable to a simple two-dimensional optimization problem, see Section 5.2, and regarding the computation of the expectations we derive analytical expressions using the inverse-probit approximation of the logistic function, see Section 5.1. For linear regression we show in the next section the implicit equations can be rewritten as an explicit scheme.

\section{Links with the Kalman filter}

In the previous section, we have developed the R-VGA update equations and have shown these updates resemble the averaged version of the online Newton scheme. We now describe in more detail this connection and show there is indeed an algebraic equivalence between the R-VGA scheme and the online Newton scheme in the linear case, or the nonlinear case under linearization assumptions. We establish also a connection with other second-order online algorithms like the Kalman filter or the natural gradient.

Let the stochastic loss function index at $t$ be defined as:

$$
\ell_{t}(\theta)=-\log p\left(y_{t} \mid \theta\right) .
$$

As before we suppose the observations to be independent conditionally on $\theta$ such that the likelihood writes $\log p\left(Y_{t} \mid \theta\right)=\sum_{i=1}^{t} \log p\left(y_{i} \mid \theta\right)$, so that the loss up to $t$ shall be defined as:

$$
L_{t}(\theta)=\sum_{i=1}^{t} \ell_{i}(\theta)
$$

\subsection{The linear regression case}

We now assume the observations are Gaussian and are linearly related to $\theta$ such that $p\left(y_{t} \mid \theta\right)=\mathscr{N}\left(H_{t} \theta, R_{t}\right)$. In this case, the least mean squares cost function (33) for $t$ observations writes:

$$
\begin{aligned}
L_{t}(\theta)=\sum_{i=1}^{t} \ell_{i}(\theta) & =\frac{1}{2} \theta^{T}\left(\sum_{i=1}^{t} H_{i}^{T} R_{i}^{-1} H_{i}\right) \theta-\left(\sum_{i=1}^{t} y_{i} R_{i}^{-1} H_{i}\right) \theta+\frac{1}{2} \sum_{i=1}^{t} y_{i}^{T} R_{i}^{-1} y_{i} \\
& =\frac{1}{2} \theta^{T} Q_{t} \theta-v_{t}^{T} \theta+C, \quad \text { where we let } Q_{t}=\sum_{i=1}^{t} H_{i}^{T} R_{i}^{-1} H_{i},
\end{aligned}
$$


where $C$ is a constant with respect to $\theta$. Assuming $Q_{t}$ is invertible and the solution to the least mean squares problem is given by the normal equation $\theta_{t}^{*}=Q_{t}^{-1} v_{t}$, we can express $\theta_{t}^{*}$ as a function of $\theta_{t-1}^{*}$, and we then recover the linear Kalman filter or equivalently the online Newton algorithm.

We prove now that in the simple case of linear regression the R-VGA updates of Theorem 1 are strictly equivalent to the linear Kalman filter and the online Newton algorithm. This is logical, as the posteriors are then Gaussian so that the KL divergence may be made equal to 0 at each step.

Theorem 2. We suppose the observation model is Gaussian and linear $y_{t}=H_{t} \theta_{t}+\varepsilon_{t}$, with $\varepsilon_{t} \sim \mathscr{N}\left(0, R_{t}\right)$, and the prior is defined as $p_{0}=q_{0}=\mathscr{N}\left(\mu_{0}, P_{0}\right)$ and we let $Q_{0}=P_{0}^{-1}$. Then, the $R$-VGA equations (3)-(4):

\section{$R-V G A$}

$$
\begin{aligned}
& \mu_{t}=\mu_{t-1}-P_{t-1} \mathbf{E}_{q_{t}}\left[\nabla_{\theta} \ell_{t}(\theta)\right] \\
& P_{t}^{-1}=P_{t-1}^{-1}+\mathbf{E}_{q_{t}}\left[\nabla_{\theta}^{2} \ell_{t}(\theta)\right],
\end{aligned}
$$

are algebraically equivalent to the explicit scheme:

\section{R-VGA (explicit scheme)}

$$
\begin{aligned}
& \mu_{t}=\mu_{t-1}-P_{t} \mathbf{E}_{q_{t-1}}\left[\nabla_{\theta} \ell_{t}(\theta)\right] \\
& P_{t}^{-1}=P_{t-1}^{-1}+\mathbf{E}_{q_{t-1}}\left[\nabla_{\theta}^{2} \ell_{t}(\theta)\right],
\end{aligned}
$$

and to the online Newton descent defined as:

\section{Online Newton descent}

$$
\begin{aligned}
\mu_{t} & =\mu_{t-1}-Q_{t}^{-1} \nabla \ell_{t}\left(\mu_{t-1}\right) \\
Q_{t} & =Q_{t-1}+\nabla^{2} \ell_{t}\left(\mu_{t-1}\right),
\end{aligned}
$$

as well as to the linear Kalman filter for a static state $\theta$ defined by:

$$
\begin{aligned}
& \text { Kalman filter } \\
& \begin{array}{l}
\mu_{t}=\mu_{t-1}+K_{t}\left(y_{t}-H_{t} \mu_{t-1}\right) \\
K_{t}=P_{t-1} H_{t}^{T}\left(R_{t}+H_{t} P_{t-1} H_{t}^{T}\right)^{-1} \\
P_{t}=\left(\mathbb{I}-K_{t} H_{t}\right) P_{t-1} .
\end{array}
\end{aligned}
$$

They all correspond to the optimal iterative algorithm for the least mean squares problem weighted by $R_{t}$. From a probabilistic viewpoint it means at each t the computed parameters satisfy $\mathscr{N}\left(\theta \mid \mu_{t}, P_{t}\right) \sim p(\theta \mid$ $\left.y_{1}, \ldots, y_{t}\right)$.

The full proof is given in Appendix 9.2. The equivalence between the Kalman filter and online Newton is already well-known, see Bertsekas [1996], but the equivalence with our variational approach is novel. Regarding this point, we note that the Kalman filter update (42) is known to write in information form $P_{t}^{-1}=P_{t-1}^{-1}+H_{t} R_{t}^{-1} H_{t}$ hence we immediately recover (36). Regarding (35), it is easily shown to rewrite in our context $\mu_{t}=\mu_{t-1}+P_{t-1} H_{t}^{T} R_{t}^{-1}\left(y_{t}-H_{t} \mu_{t}\right)$. Although the latter bears a resemblance to (40), the link is not straightforward and relies on the nontrivial but useful fact that one may rewrite the Kalman gain as $K_{t}=P_{t} H_{t}^{T} R_{t}^{-1}$.

The theorem shows how the issues inherent to the implicit scheme evaporate in the linear case, as we may arrive at expectations that involve the known distribution $q_{t-1}$. Moreover, it shows the algorithm is unbeatable in the linear Gaussian case: this is logical as it outputs a Gaussian that minimizes the KL divergence to a constant times $p\left(y_{t} \mid \theta\right) q_{t-1}(\theta)$, but the latter is Gaussian in the present case, so that the algorithm exactly recovers the partial posterior at each step. 


\subsection{Application to exponential families}

When the likelihood stems from an exponential family ${ }^{1}$, we may proceed along the same lines under linearization assumptions. This way, we may extend the results by Ollivier [2018] about the equivalence between the natural gradient and the extended Kalman filter (EKF) to a full equivalence with the proposed R-VGA for a linearized model. We consider in this section that the observations follow an exponential family distribution with a nonlinear model $h$, that is,

$$
\begin{aligned}
& p(y \mid \theta)=m(y) \exp \left(\eta(\theta)^{T} y-A(\eta(\theta))\right) \\
& \mathbf{E}(y \mid \theta):=\bar{y}=h(\theta) \text { where } h \text { is the model, } \\
& \eta=g(\bar{y})=g(h(\theta)) \text { where } g \text { is the link function. }
\end{aligned}
$$

Assume now that at each step $t$ of an online algorithm, the model $h$ is approximated through a first-order Taylor expansion around the previous estimate $\mu_{t-1}$ :

$$
h(\theta) \approx h\left(\mu_{t-1}\right)+\nabla_{\theta}^{T} h\left(\mu_{t-1}\right)\left(\theta-\mu_{t-1}\right)=h\left(\mu_{t-1}\right)+H_{t}\left(\theta-\mu_{t-1}\right),
$$

and the covariance is evaluated around the last mean $h\left(\mu_{t-1}\right)$ and assumed independent of $\theta$ :

$$
\operatorname{Cov}\left(y_{t}\right)=\mathbf{E}\left[\left(y_{t}-h\left(\mu_{t-1}\right)\right)\left(y_{t}-h\left(\mu_{t-1}\right)\right)^{T}\right]:=R_{t} .
$$

From the property of exponential families, the mean vector and the covariance matrix are deduced from the first and second derivative of the $\log$-partition function $A(\eta)$, so we have the following relations:

$$
\begin{aligned}
& \frac{\partial A\left(\eta_{t}\right)}{\partial \eta_{t}}=\bar{y}_{t}=h\left(\mu_{t-1}\right)+H_{t}\left(\theta-\mu_{t-1}\right) \\
& \text { and } \frac{\partial^{2} A\left(\eta_{t}\right)}{\partial \eta_{t}^{2}}=\frac{\partial \bar{y}_{t}}{\partial \eta_{t}}=R_{t} .
\end{aligned}
$$

The loss function under the latter linearization writes:

$$
\tilde{\ell}_{t}(\theta)=-\log p\left(y_{t} \mid \theta\right)=-\eta_{t}^{T} y_{t}+A\left(\eta_{t}\right)+C
$$

and its derivatives are approximated as:

$$
\begin{aligned}
& \nabla_{\theta} \tilde{\ell}_{t}(\theta)=\frac{\partial \tilde{\ell}_{t}}{\partial \eta} \frac{\partial \eta}{\partial \bar{y}} \frac{\partial \bar{y}}{\partial \theta}=-H_{t}^{T} R_{t}^{-1}\left(y_{t}-h\left(\mu_{t-1}\right)-H_{t}\left(\theta-\mu_{t-1}\right)\right) \\
& \nabla_{\theta}^{2} \tilde{\ell}_{t}(\theta)=H_{t}^{T} R_{t}^{-1} H_{t} .
\end{aligned}
$$

We can then rewrite the update equation (4) as:

$$
\begin{aligned}
P_{t}^{-1} & =P_{t-1}^{-1}+\mathbf{E}_{q_{t}}\left[H_{t}^{T} R_{t}^{-1} H_{t}\right] \\
& =P_{t-1}^{-1}+H_{t}^{T} R_{t}^{-1} H_{t},
\end{aligned}
$$

and we find the same form that as in the linear case, and similar derivations may be applied to the update equation (3). Combined with the results from Ollivier [2018] we finally find:

\footnotetext{
${ }^{1}$ Note that this is different from considering the variational distribution within an exponential family, as done at the end of Section 3.1 .
} 
Theorem 3. For observations based on an exponential family (43), under the approximations and assumptions (44)-(45), that is, using the equalities (47)-(48), one may prove that

\section{The linearized R-VGA}

$$
\begin{aligned}
& \mu_{t}=\mu_{t-1}-P_{t-1} \mathbf{E}_{q_{t}}\left[\nabla_{\theta} \tilde{\ell}_{t}(\theta)\right] \\
& P_{t}^{-1}=P_{t-1}^{-1}+\mathbf{E}_{q_{t}}\left[\nabla_{\theta}^{2} \tilde{\ell}_{t}(\theta)\right],
\end{aligned}
$$

is algebraically equivalent to the explicit scheme :

$$
\begin{aligned}
& \text { The linearized } \boldsymbol{R} \text {-VGA (explicit scheme) } \\
& \mu_{t}=\mu_{t-1}-P_{t} \mathbf{E}_{q_{t-1}}\left[\nabla_{\theta} \tilde{\ell}_{t}(\theta)\right] \\
& P_{t}^{-1}=P_{t-1}^{-1}+\mathbf{E}_{q_{t-1}}\left[\nabla_{\theta}^{2} \tilde{\ell}_{t}(\theta)\right]
\end{aligned}
$$

and to the online natural gradient on the exact loss $\ell_{t}$ defined as:

The online natural gradient with learning rate $\frac{1}{t+1}$

given $\mu_{0}$ and $J_{0}$ supposed invertible:

$$
\begin{aligned}
& \mu_{t}=\mu_{t-1}-\left.\frac{1}{t+1} J_{t}^{-1} \nabla_{\theta} \ell_{t}(\theta)\right|_{\mu_{t-1}} \\
& J_{t}=\frac{t}{t+1} J_{t-1}+\left.\frac{1}{t+1} \mathbf{E}_{y}\left[\nabla_{\theta}^{2} \ell_{t}(\theta)\right]\right|_{\mu_{t-1}},
\end{aligned}
$$

as well as to the extended Kalman filter for a static state:

\section{The extended Kalman filter}

given $\mu_{0}$ and $P_{0}$ supposed invertible:

$$
\begin{aligned}
& \mu_{t}=\mu_{t-1}+K_{t}\left(y_{t}-h\left(\mu_{t-1}\right)\right) \\
& H_{t}=\nabla_{\theta}^{T} h\left(\mu_{t-1}\right) \\
& K_{t}=P_{t-1} H_{t}^{T}\left(R_{t}+H_{t} P_{t-1} H_{t}^{T}\right)^{-1} \\
& P_{t}^{-1}=P_{t-1}^{-1}+H_{t}^{T} R_{t}^{-1} H_{t},
\end{aligned}
$$

where the last line may alternatively be re-written $P_{t}=\left(\mathbb{I}-K_{t} H_{t}\right) P_{t-1}$.

The full proof has been moved to Appendix 9.3.

As for the linear case, it is shown in the proof in Appendix 9.3 that the implicit scheme is equivalent to the explicit scheme. However it is only true for the linearized version of the R-VGA. The equivalence between explicit and implicit scheme is lost for the exact R-VGA version.

Moreover the theorem shows that, for observations following an exponential family, all the mentioned algorithms including ours coincide under linearization assumptions. In particular, the natural gradient and the EKF appear as a particular case of the R-VGA where the loss function is linearized. This is important, though, as experiments of Section 6 will demonstrate that for the logistic loss the non linearized R-VGA outperforms the EKF, and equivalently the online natural gradient.

Remark 1. The online natural gradient, in Equation (54), uses an expectation under y whereas the exact $R$-VGA uses an expectation under $\theta$. These expectations have nothing in common. For the natural gradient the expectation comes from the Fisher matrix definition and allows for the Gauss-Newton form for the Hessian. 
Indeed, the Gauss-Newton form is equivalent to the Hessian form only if we average under the observations $y$ : $\mathbf{E}_{y}\left[\nabla_{\theta} \ell_{t}(\theta) \times \nabla_{\theta} \ell_{t}(\theta)\right]=\mathbf{E}_{y}\left[\nabla_{\theta}^{2} \ell_{t}(\theta)\right]$, otherwise the Hessian can be strongly biased as shown by Kunstner et al. [2019]. For a GLM model (or a linearized model as in Theorem 3), we can drop the expectation $\mathbf{E}_{y}$ without loosing precision if we introduce the covariance matrix $R_{t}: \mathbf{E}_{y}\left[\nabla_{\theta}^{2} \ell_{t}(\theta)\right]=\nabla_{\theta} h(\theta) \times R_{t}^{-1} \times \nabla_{\theta} h(\theta)$ as shown by Martens [2020] in the generalized Gauss-Newton framework. The detailed proof of this relation is recalled at the end of Appendix 9.3.

\section{Application to logistic regression}

In this section, we apply the R-VGA to binary classification. We compare it to the extended Kalman filter and to the quadratic Kalman filter which is a variational variant using an upper bound on the logistic loss. In logistic regression, the observation are binary labels $y_{t} \in\{0,1\}$ associated to input variable $x_{t} \in \mathbf{R}^{d}$. The loss relies on the logistic function $\sigma(x)=\frac{1}{1+\exp (-x)}$ as:

$$
\ell_{t}(\theta)=-\log p\left(y_{t} \mid \theta\right)=-y_{t} \log \sigma\left(x_{t}^{T} \theta\right)-\left(1-y_{t}\right) \log \left(1-\sigma\left(x_{t}^{T} \theta\right)\right) .
$$

\subsection{The R-VGA for logistic regression}

We need first to compute the expectations which appear in the R-VGA. Using the relation $\sigma^{\prime}=(1-\sigma) \sigma$, the first and second derivatives of the logistic loss are given by:

$$
\begin{aligned}
& \nabla_{\theta} \ell_{t}(\theta)=-\left(y_{t}-\sigma\left(x_{t}^{T} \theta\right)\right) x_{t}, \\
& \nabla_{\theta}^{2} \ell_{t}(\theta)=x_{t} \sigma^{\prime}\left(x_{t}^{T} \theta\right) x_{t}^{T} .
\end{aligned}
$$

The Gaussian expectations of these derivatives read:

$$
\begin{aligned}
& \mathbf{E}_{q_{t}}\left[\nabla_{\theta} \ell_{t}(\boldsymbol{\theta})\right]=-y_{t} x_{t}+\mathbf{E}_{q_{t}}\left[\boldsymbol{\sigma}\left(x_{t}^{T} \boldsymbol{\theta}\right)\right] x_{t} \\
& \mathbf{E}_{q_{t}}\left[\nabla_{\theta}^{2} \ell_{t}(\boldsymbol{\theta})\right]=x_{t} \mathbf{E}_{q_{t}}\left[\boldsymbol{\sigma}^{\prime}\left(x_{t}^{T} \boldsymbol{\theta}\right)\right] x_{t}^{T} .
\end{aligned}
$$

To compute $\mathbf{E}_{q_{t}}\left[\sigma\left(x_{t}^{T} \theta\right)\right]$, we use the marginalized variable $a$ along the vector $x_{t}$, i.e., $a=x_{t}^{T} \theta$, with distribution $\mathscr{N}\left(\mu_{a}, \sigma_{a}^{2}\right)$ where $\mu_{a}=x_{t}^{T} \mu_{t}$ and $\sigma_{a}^{2}=x_{t}^{T} P_{t} x_{t}$, as proposed by Barber and Bishop [1998]. Then we deduce:

$$
\mathbf{E}_{q_{t}}\left[\sigma\left(x_{t}^{T} \theta\right)\right]=\int \sigma\left(x_{t}^{T} \theta\right) q_{t}(\theta) d \theta=\int_{-\infty}^{+\infty} \sigma(a) p(a) d a
$$

For analytical tractability we approximate the logistic function with the inverse probit function as proposed by Barber and Bishop [1998]:

$$
\begin{aligned}
& \sigma(a) \approx \Phi(\lambda a):=\frac{1}{2}\left(1+\operatorname{erf}\left(\frac{\lambda a}{\sqrt{2}}\right)\right) \\
& \text { with } \operatorname{erf}(x)=\frac{2}{\sqrt{\pi}} \int_{0}^{x} \exp \left(-u^{2}\right) d u \text { and } \lambda=\sqrt{\frac{\pi}{8}} .
\end{aligned}
$$

Figure 1 shows a good match, indicating this choice well approximated the logistic function. 
Approximation of the sigmoid function and its derivative
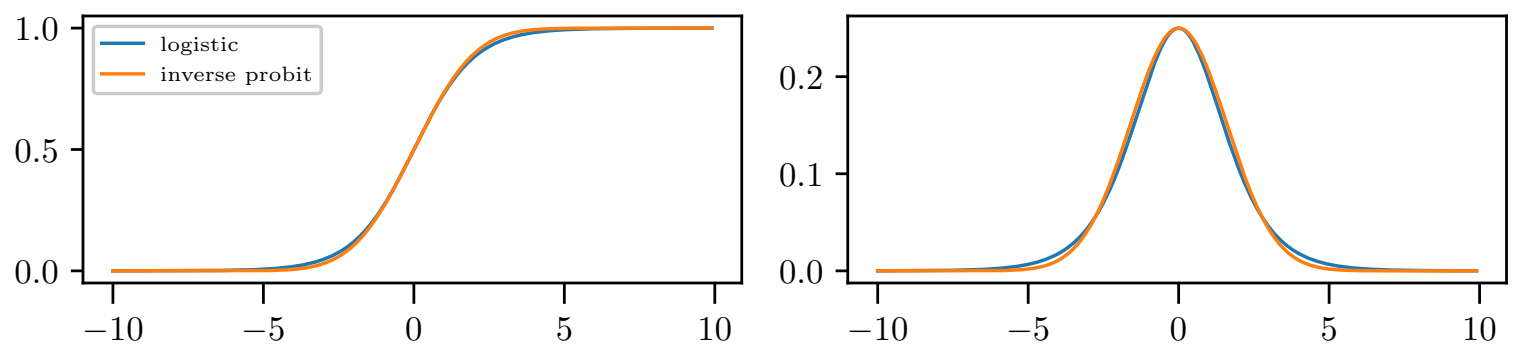

Figure 1: Comparison of the logistic function and the inverse probit function (left) ands their derivatives (right). Both match well, and shall be indifferently used in applications.

We obtain analytical expressions for the expectations:

$$
\begin{aligned}
& \int_{-\infty}^{+\infty} \sigma(a) \mathscr{N}\left(a \mid \mu_{a}, \sigma_{a}^{2}\right) d a \approx \int_{-\infty}^{+\infty} \int_{-\infty}^{\lambda a} \mathscr{N}(x \mid 0,1) \mathscr{N}\left(a \mid \mu_{a}, \sigma_{a}^{2}\right) d x d a \\
& =\int_{-\infty}^{+\infty} \int_{-\infty}^{0} \mathscr{N}\left(x \mid-a, \lambda^{-2}\right) \mathscr{N}\left(a \mid \mu_{a}, \sigma_{a}^{2}\right) d x d a=\int_{-\infty}^{0}\left[\mathscr{N}\left(0, \lambda^{-2}\right) * \mathscr{N}\left(-\mu_{a}, \sigma_{a}^{2}\right)\right](x) d x \\
& =\int_{-\infty}^{0} \mathscr{N}\left(x \mid-\mu_{a}, \sigma_{a}^{2}+\lambda^{-2}\right) d x=\Phi\left(\frac{\mu_{a}}{\sqrt{\sigma_{a}^{2}+\lambda^{-2}}}\right) \approx \sigma\left(\frac{\mu_{a}}{\lambda \sqrt{\sigma_{a}^{2}+\lambda^{-2}}}\right) .
\end{aligned}
$$

To compute $\mathbf{E}_{q_{t}}\left[\sigma^{\prime}\left(x_{t}^{T} \theta\right)\right]$, we follow Daunizeau [2017] where we let $\beta=\lambda^{-1}=\sqrt{8 / \pi}$ :

$$
\begin{aligned}
& \int_{-\infty}^{+\infty} \sigma^{\prime}(a) p(a) d a \approx \int_{-\infty}^{+\infty} \mathscr{N}\left(a \mid 0, \beta^{2}\right) p(a) d a=\frac{1}{\beta \sigma_{a} 2 \pi} \int_{-\infty}^{+\infty} \exp -\frac{1}{2}\left(-\frac{a^{2}}{\beta^{2}}-\frac{\left(a-\mu_{a}\right)^{2}}{\sigma_{a}^{2}}\right) d a \\
& =\frac{1}{\beta \sigma_{a} 2 \pi} \exp \left(-\frac{1}{2} \frac{\mu_{a}^{2}}{\sigma_{a}^{2}+\beta^{2}}\right) \int_{-\infty}^{+\infty} \exp \left(-\frac{1}{2} \frac{\sigma_{a}^{2}+\beta^{2}}{\sigma_{a}^{2} \beta^{2}}\left(a-\frac{\mu_{a} \beta^{2}}{\sigma_{a}^{2}+\beta^{2}}\right)^{2}\right) d a \\
& =\frac{1}{\beta \sigma_{a} 2 \pi} \exp \left(-\frac{1}{2} \frac{\mu_{a}^{2}}{\sigma_{a}^{2}+\beta^{2}}\right) \sqrt{\frac{2 \pi \sigma_{a}^{2} \beta^{2}}{\sigma_{a}^{2}+\beta^{2}}}=\frac{1}{\sqrt{2 \pi\left(\sigma_{a}^{2}+\beta^{2}\right)}} \exp \left(-\frac{1}{2} \frac{\mu_{a}^{2}}{\sigma_{a}^{2}+\beta^{2}}\right) \\
& =\mathscr{N}\left(0 \mid \mu_{a}, \sigma_{a}^{2}+\beta^{2}\right) \approx \frac{\beta}{\sqrt{\sigma_{a}^{2}+\beta^{2}}} \sigma^{\prime}\left(\frac{\mu_{a} \beta}{\sqrt{\sigma_{a}^{2}+\beta^{2}}}\right) .
\end{aligned}
$$

Coming back to (56)-(57), we find:

$$
k_{t}=\frac{\beta}{\sqrt{x_{t}^{T} P_{t} x_{t}+\beta^{2}}}, \quad \mathbf{E}_{q_{t}}\left[\nabla_{\theta} \ell_{i}(\theta)\right] \approx-y_{t} x_{t}+\sigma\left(k_{t} x_{t}^{T} \mu_{t}\right) x_{t}, \quad \mathbf{E}_{q_{t}}\left[\nabla_{\theta}^{2} \ell_{t}(\theta)\right] \approx x_{t} k_{t} \sigma^{\prime}\left(k_{t} x_{t}^{T} \mu_{t}\right) x_{t}^{T} .
$$

As shown by Figure 1, the inverse probit approximation is a very good approximation. Gathering those results, the R-VGA now reads

$$
\begin{aligned}
& k_{t}=\frac{\beta}{\sqrt{x_{t}^{T} P_{t} x_{t}+\beta^{2}}} \\
& \mu_{t}=\mu_{t-1}+P_{t-1} x_{t}\left(y_{t}-\sigma\left(k_{t} x_{t}^{T} \mu_{t}\right)\right), \\
& P_{t}^{-1}=P_{t-1}^{-1}+k_{t} \sigma^{\prime}\left(k_{t} x_{t}^{T} \mu_{t}\right) x_{t} x_{t}^{T} .
\end{aligned}
$$


We see we have obtained analytical expressions for the integrals involved, as in the linear case. However, the algorithm remains implicitly defined, and we will shortly show how to solve the problem. A prediction based on any obtained distribution $q \sim \mathscr{N}(\mu, P)$, for an input $x_{s}$ may then be computed as:

$$
\begin{aligned}
& \hat{y}_{s}=\mathbf{E}_{q}\left[\sigma\left(x_{s}^{T} \theta\right)\right]=\sigma\left(k_{s} x_{s}^{T} \mu\right) \\
& \text { where } k_{s}=\frac{\beta}{\sqrt{x_{s}^{T} P x_{s}+\beta^{2}}} .
\end{aligned}
$$

The variance of the prediction may also be computed as (following Daunizeau [2017]):

$$
\operatorname{Var}\left(\hat{y}_{s}\right)=\mathbf{E}_{q}\left[\sigma^{2}\left(x_{s}^{T} \theta\right)\right]-\mathbf{E}_{q}^{2}\left[\sigma\left(x_{s}^{T} \theta\right)\right]=\sigma\left(k_{s} x_{s}^{T} \mu\right)\left(1-\sigma\left(k_{s} x_{s}^{T} \mu\right)\right)\left(1-k_{s}\right) .
$$

Further details about output prediction and uncertainty assessment of the R-VGA may be found in Appendix 9.4.

\subsection{Solving the implicit scheme}

Solving the implicit scheme (60)-(61) is amenable to a two-dimensional fixed point problem. Indeed, we see that as soon as the scalar quantities $x_{t}^{T} \mu_{t}$ and $x_{t}^{T} P_{t-1} x_{t}$ are known the scheme becomes explicit. To find those quantities, we consider the following change of variables:

$$
\begin{aligned}
& \text { unknown variables } \\
& \begin{array}{l}
\alpha=x_{t}^{T} \mu_{t} \\
v=x_{t}^{T} P_{t} x_{t}
\end{array} \\
& \text { parameters in the equa } \\
& \alpha_{0}=x_{t}^{T} \mu_{t-1} \\
& v_{0}=x_{t}^{T} P_{t-1} x_{t} .
\end{aligned}
$$$$
\text { parameters in the equations }
$$

We also rewrite $k_{t}$ in (59) as $k(v)$ to make the dependency clearly appear. By multiplying equation (60) by $x_{t}^{T}$ on the left we find:

$$
\alpha=-v_{0} \sigma(\alpha k(v))+\alpha_{0}+v_{0} y_{t} .
$$

Applying the Woodbury formula to (61) we obtain:

$$
P_{t}=P_{t-1}-P_{t-1} x_{t}\left(\frac{1}{k(v) \sigma^{\prime}\left(k(v) x_{t}^{T} \mu_{t}\right)}+x_{t}^{T} P_{t-1} x_{t}\right)^{-1} x_{t}^{T} P_{t-1}
$$

Multiplying this equation by $x_{t}^{T}$ on the left and $x_{t}$ on the right we find:

$$
v=v_{0}-v_{0}^{2}\left(\frac{1}{k(v) \sigma^{\prime}(\alpha k(v))}+v_{0}\right)^{-1}=\frac{v_{0}}{1+v_{0} k(v) \sigma^{\prime}(\alpha k(v))} .
$$

We end up with to a two-dimensional implicit scheme (64)-(66). To solve it we need to find the roots of $F_{\alpha_{0}, v_{0}, y}$ defined as:

$$
\begin{aligned}
F_{\alpha_{0}, v_{0}, y}: \mathbb{R}^{2} & \rightarrow \mathbb{R}^{2} \\
(\alpha, v) & \rightarrow(f(\alpha, v), g(\alpha, v)),
\end{aligned}
$$


with:

$$
\begin{aligned}
& f(\alpha, v)=\alpha+v_{0} \sigma(\alpha k(v))-\alpha_{0}-v_{0} y \\
& g(\alpha, v)=v-\frac{v_{0}}{1+v_{0} k(v) \sigma^{\prime}(\alpha k(v))}
\end{aligned}
$$

on the domain:

$$
\begin{aligned}
& \alpha_{0}+v_{0}(y-1) \leq \alpha \leq \alpha_{0}+v_{0} y \\
& 0 \leq v_{0}\left(1-\frac{v_{0}}{4+v_{0}}\right) \leq v \leq v_{0} .
\end{aligned}
$$

If need be, the domain may be further reduced, as shown in Appendix 9.5, where the shape of the functions $f$ and $g$ are also displayed, in Figure 12. To find the roots of $F_{\alpha_{0}, v_{0}, y}$, we have opted for the hybrid-Powel method [Powell, 2010] which converges quite fast for relatively moderate values of $\alpha_{0}$ and $v_{0}$ (typically below $10^{-4}$ ). To enforce the fact that $v$ be positive, we change variables in the optimization scheme letting $\exp (\tilde{\boldsymbol{v}})=\boldsymbol{v}$.

We obtain finally the following implicit updates:

\section{R-VGA (implicit) for logistic regression}

$$
\begin{aligned}
& \alpha_{0}=x_{t}^{T} \mu_{t-1}, v_{0}=x_{t}^{T} P_{t-1} x_{t}, \\
& \alpha, v \leftarrow \text { roots of } F_{\alpha_{0}, v_{0}, y_{t}} \text { defined in }(67)-(70), \\
& k_{t}=\frac{\beta}{\sqrt{v+\beta^{2}}}, \\
& \mu_{t}=\mu_{t-1}+P_{t-1} x_{t}\left(y_{t}-\sigma\left(k_{t} \alpha\right)\right), \\
& P_{t}=P_{t-1}-P_{t-1} x_{t} x_{t}^{T} P_{t-1} /\left(\frac{1}{k_{t} \sigma^{\prime}\left(k_{t} \alpha\right)}+x_{t}^{T} P_{t-1} x_{t}\right) .
\end{aligned}
$$

\subsection{Numerical complexity}

To avoid inverting $P_{t}$ as in (61), we have used Woodbury's formula in (77) to update $P_{t}$ directly, as in the Kalman filter, along the lines of (65). At each step we see $O\left(d^{2}\right)$ operations are required, as soon as the quantities $x_{t}^{T} \mu_{t}$ and $x_{t}^{T} P_{t-1} x_{t}$ have been found, which amounts to computing a few iterations of a two-dimensional optimizer. This makes an overall computation of cost of order $O\left(d^{2} N\right)$.

Note that, if one wants to enforce the positivity of matrix $P_{t}$, it is possible without increasing the overall computational cost to resort to a square-root implementation akin to the Kalman filter's, see Bierman [1975].

\subsection{Alternative algorithms}

The proposed R-VGA algorithm is optimal at each step given the previous Gaussian approximation, that is, it minimizes the divergence between $q_{t}(\theta)$ and $p\left(y_{t} \mid \theta\right) q_{t-1}(\theta)$ assuming the inverse probit approximation of the logistic function. It may be compared with other variants, that is, our explicit scheme approximation, the well-known extended Kalman filter applied to logistic regression as presented by Ollivier [2018] and an online version of the variational approach to logistic regression of Jaakkola and Jordan [1997] we introduced hereafter. 


\subsubsection{The explicit scheme approximation}

Our approximated explicit-scheme based R-VGA (30)-(31) may be readily applied using the analytical expressions (58) for the integrals replacing $\mu_{t}, P_{t}$ with $\mu_{t-1}, P_{t-1}$ :

$$
\begin{aligned}
& \text { R-VGA (explicit) for logistic regression } \\
& k_{t}=\frac{\beta}{\sqrt{x_{t}^{T} P_{t-1} x_{t}+\beta^{2}}} \\
& \mu_{t}=\mu_{t-1}+P_{t} x_{t}\left(y_{t}-\sigma\left(k_{t} x_{t}^{T} \mu_{t-1}\right)\right), \\
& P_{t}=P_{t-1}-P_{t-1} x_{t} x_{t}^{T} P_{t-1} /\left(\frac{1}{k_{t} \sigma^{\prime}\left(k_{t} x_{t}^{T} \mu_{t-1}\right)}+x_{t}^{T} P_{t-1} x_{t}\right) .
\end{aligned}
$$

Albeit optimal in the context of linear regression, this scheme is no longer optimal in the non-linear case. However, We find in the sequel its performance is often close to the implicit scheme's, and better than the EKF's for the logistic regression problem. Comparisons are displayed in our experiments indeed.

\subsubsection{The extended Kalman filter for logistic regression}

The extended Kalman filter (EKF) is based on the linearization of the likelihood, and is equivalent to the natural gradient for the logistic regression, see Ollivier [2018]. We have shown in Theorem 3 that the extended Kalman filter is also equivalent to the linearized version of the R-VGA. Comparing both will indicate how the exact version behaves compared to the linearized one. To build the extended Kalman filter we need to compute the covariance of the observations $R_{t}$ and the observation matrix $H_{t}$ which is the Jacobian of the averaged observed value at the previous estimate. Following Ollivier [2018], those quantities write:

$$
\begin{aligned}
& R_{t}=\operatorname{Cov}\left(y_{t}\right)=\sigma^{\prime}\left(x_{t}^{T} \mu_{t-1}\right)=\sigma\left(x_{t}^{T} \mu_{t-1}\right)\left(1-\sigma\left(x_{t}^{T} \mu_{t-1}\right)\right) \\
& \bar{y}_{t}=\sigma\left(x_{t}^{T} \theta\right) \approx \sigma\left(\mu_{t-1}\right)+H_{t}\left(\theta-\mu_{t-1}\right) \\
& \text { where } H_{t}=\frac{\partial \sigma\left(x_{t}^{T} \theta\right)}{\partial \theta}\left(\mu_{t-1}\right)=\sigma^{\prime}\left(x_{t}^{T} \mu_{t-1}\right) x_{t}^{T}=R_{t} x_{t}^{T},
\end{aligned}
$$

leading to the following equations for the EKF:

$$
\begin{aligned}
& R_{t}=\sigma\left(x_{t}^{T} \mu_{t-1}\right)\left(1-\sigma\left(x_{t}^{T} \mu_{t-1}\right)\right) \\
& H_{t}=R_{t} x_{t}^{T} \\
& K_{t}=P_{t-1} H_{t}^{T}\left(R_{t}+H_{t} P_{t-1} H_{t}^{T}\right)^{-1} \\
& \mu_{t}=\mu_{t-1}+K_{t}\left(y_{t}-\sigma\left(x_{t}^{T} \mu_{t-1}\right)\right) \\
& P_{t}=\left(\mathbb{I}-K_{t} H_{t}\right) P_{t-1} .
\end{aligned}
$$

Using the fact that $K_{t}=P_{t} H_{t}^{T} R_{t}^{-1}$ and $P_{t}^{-1}=P_{t-1}^{-1}+H_{t}^{T} R_{t}^{-1} H_{t}$ allows rewriting the equations in compact form as:

The extended Kalman filter (EKF) for logistic regression

$$
\begin{aligned}
& \mu_{t}=\mu_{t-1}+P_{t} x_{t}\left(y_{t}-\sigma\left(x_{t}^{T} \mu_{t-1}\right)\right), \\
& P_{t}=P_{t-1}-P_{t-1} x_{t} x_{t}^{T} P_{t-1} /\left(\frac{1}{\sigma^{\prime}\left(x_{t}^{T} \mu_{t-1}\right)}+x_{t}^{T} P_{t-1} x_{t}\right) .
\end{aligned}
$$

The obtained formulas bear a strong resemblance to the R-VGA, but important differences shall be noted, though. Indeed, in the rightmost terms $P_{t}$ and $P_{t-1}$ are swapped and so are $\mu_{t}$ and $\mu_{t-1}$. Besides, the factor 
$0<k_{t} \leq 1$ which appears in the R-VGA is absent in the EKF. Those differences are not wholly surprising, as the extended Kalman filter linearizes the sigmoid function around the last estimate $\mu_{t-1}$ to compute the information matrix $P_{t}^{-1}$, whereas R-VGA truly minimizes the KL divergence. To this respect, the EKF may be viewed as an online version of the Laplace approximation.

\subsubsection{The quadratic Kalman filter for logistic regression}

The logistic loss has a sharp form and it is known that the Laplace approximation can make the Hessian vanish. As a remedy Jaakkola and Jordan [1997] have proposed a quadratic upper bound for the logistic loss, easier to minimize. Following their approach, we construct first a lower bound for the sigmoid using the Legendre transform, introducing local tangents of slope $\eta$ at points $\xi$ (see Jaakkola and Jordan [1997] for more details):

$$
\begin{aligned}
& \sigma(\theta) \geq \sigma(\xi) \exp \left(\frac{\theta-\xi}{2}+\eta\left(\theta^{2}-\xi^{2}\right)\right) \forall \xi \in \mathbb{R} \\
& \text { with } \eta=-\frac{1}{2 \xi}\left(\sigma(\xi)-\frac{1}{2}\right) .
\end{aligned}
$$

Given this lower bound on the sigmoid, the upper bound for $-\log p(y \mid \theta)$ has a quadratic form in $\theta$ easier to minimize than the initial logistic loss (here $x$ is the input as before):

$$
-\log p(y \mid \theta) \leq-x^{T} \theta y-\log \sigma(\xi)+\frac{x^{T} \theta+\xi}{2}-\eta\left(\left(x^{T} \theta\right)^{2}-\xi^{2}\right)=Q(\theta, \xi) .
$$

This upper bound has also been used in the context of Gaussian processes using approximation with PolyaGamma function (see Wenzel et al. [2019], Appendix A.5). The lower and upper bound shapes are displayed in Figure 2 for dimensions one and two. In the present paper we propose to apply the R-VGA (3)-(4) to
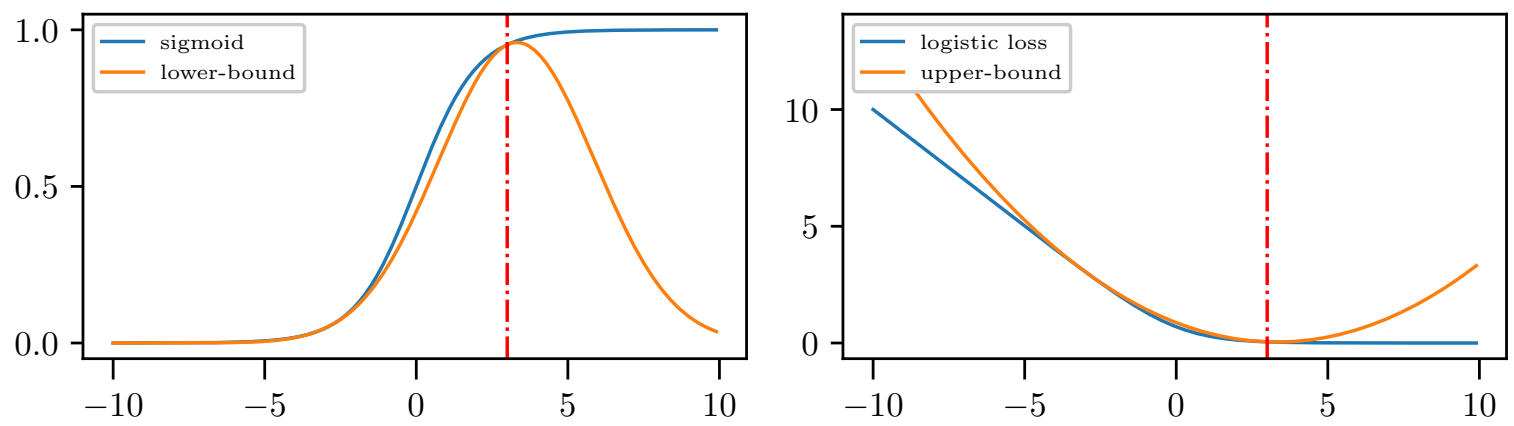

Figure 2: Quadratic lower bound on the logistic function (left) and upper bound on logistic loss (right).

$Q(\theta, \xi)$, which yields:

$$
\begin{aligned}
& \mu_{t}=\mu_{t-1}-P_{t-1} \mathbf{E}_{q_{t}}\left[\nabla_{\theta} Q(\theta, \xi)\right]=\mu_{t-1}-P_{t-1}\left(-2 \eta \mu_{t} x_{t} x_{t}^{T}+\left(\frac{1}{2}-y_{t}\right) x_{t}\right) \\
& P_{t}^{-1}=P_{t-1}^{-1}+\mathbf{E}_{q_{t}}\left[\nabla_{\theta}^{2} Q(\theta, \xi)\right]=P_{t-1}^{-1}-2 \eta x_{t} x_{t}^{T} .
\end{aligned}
$$

The simplistic form of $Q(\theta, \xi)$ allows not only to obtain analytical expressions for the integrals but also to make the update explicit. Indeed, multiplying the equality (84) by $P_{t-1}^{-1}$ on both sides, we find:

$$
\mu_{t}=\left(P_{t-1}^{-1}-2 \eta x_{t} x_{t}^{T}\right)^{-1}\left(P_{t-1}^{-1} \mu_{t-1}-\left(\frac{1}{2}-y_{t}\right) x_{t}\right)=P_{t}\left(P_{t-1}^{-1} \mu_{t-1}+\left(y_{t}-\frac{1}{2}\right) x_{t}\right),
$$


which is the same form as the one found by Jaakkola and Jordan [1997]. This expression can be further rearranged to make a "Kalman gain" appear:

$$
\begin{aligned}
& \mu_{t}=P_{t}\left(\left(P_{t}^{-1}+2 \eta x_{t} x_{t}^{T}\right) \mu_{t-1}+\left(y_{t}-\frac{1}{2}\right) x_{t}\right) \\
& =\mu_{t-1}+P_{t} x_{t}(-2 \eta)\left(\frac{y_{t}-\frac{1}{2}}{-2 \eta}-x_{t}^{T} \mu_{t-1}\right):=\mu_{t-1}+K_{t}\left(\frac{y_{t}-\frac{1}{2}}{-2 \eta}-x_{t}^{T} \mu_{t-1}\right) .
\end{aligned}
$$

This filter is closely related to the variational Kalman filter proposed for a dynamical state in Sykacek and Roberts [2003]. To distinguish it from our R-VGA filter, which is based on a different variational approach, we call it the quadratic Kalman filter (QKF) in the present paper. This filter will be benchmarked in the experiments, and compared to the proposed R-VGA. Using Woodbury formulas and the Kalman filter formalism, we obtain the following update equations:

The quadratic Kalman filter (QKF) for logistic regression

$$
\begin{aligned}
& H_{t}=x_{t}^{T} \\
& \xi_{t}^{2}=H_{t}\left(P_{t-1}+\mu_{t-1} \mu_{t-1}^{T}\right) H_{t}^{T} \\
& R_{t}^{-1}=-2 \eta=\frac{1}{\xi_{t}}\left(\frac{1}{1+e^{-\xi_{t}}}-\frac{1}{2}\right) \\
& K_{t}=P_{t-1} H_{t}^{T}\left(R_{t}+H_{t} P_{t-1} H_{t}^{T}\right)^{-1} \\
& \mu_{t}=\mu_{t-1}+K_{t}\left(R_{t}\left(y_{t}-\frac{1}{2}\right)-H_{t} \mu_{t-1}\right) \\
& P_{t}=\left(\mathbb{I}-K_{t} H_{t}\right) P_{t-1},
\end{aligned}
$$

where the update equation for $\xi$ used in (89) is derived from Jaakkola and Jordan [1997] and can also be found by differentiating the KL divergence with respect to $\xi$.

\section{Numerical experiments for logistic regression}

We consider $N$ synthetic inputs $x_{i}$ equally distributed along two Gaussians with identical covariance matrix such that the distribution of the outputs $p\left(y_{i} \mid x_{i}\right)$ writes as a sigmoid function. Moreover we suppose the mean vectors are symmetric $\mu_{1}=-\mu_{2}$, so that the linear separator passes through the origin and the constant term is null. The mean $\mu_{1}$ and $\mu_{2}$ of the Gaussian are separated by a distance $s=\left\|\mu_{1}-\mu_{2}\right\|=\left\|2 \mu_{1}\right\|$ which is a free parameter. The algorithms are initialized with a prior distribution $q_{0}=\mathscr{N}\left(\mu_{0}, \sigma_{0}^{2} \mathbf{I}\right)$. To make the problem more challenging, we consider ill-conditioned covariance matrices of the form:

$$
C=M^{T} \operatorname{Diag}\left(1,1 / 2^{c}, \ldots, 1 / d^{c}\right) M,
$$

where $M$ is a random unitary orthogonal matrix to ensure the covariance is not aligned with the axes and $c$ is a free parameter. If $c=0$ the covariance is "isotropic", otherwise it is "ill-conditioned". Moreover, we normalize the random inputs vectors for each classes $i$ such that $x_{i} \leftarrow \mu_{i}+\left(x_{i}-\mu_{i}\right) /\left\|s t d\left(x_{i}\right)\right\|$ assuming the data can be pre-processed. This allows for a better management of the separability $s$ of the dataset since the norm of the inputs scale as $\sqrt{d}$ (in the "isotropic" case).

\subsection{Comparison metrics computation}

To assess the performance of the algorithms, we propose to compute the KL divergence between the posterior and its approximations over time. To do so we note the posterior may be written $p\left(\theta \mid Y_{N}\right)=\frac{\tilde{p}\left(\theta \mid Y_{N}\right)}{Z_{p}}$ where $Z_{p}$ 

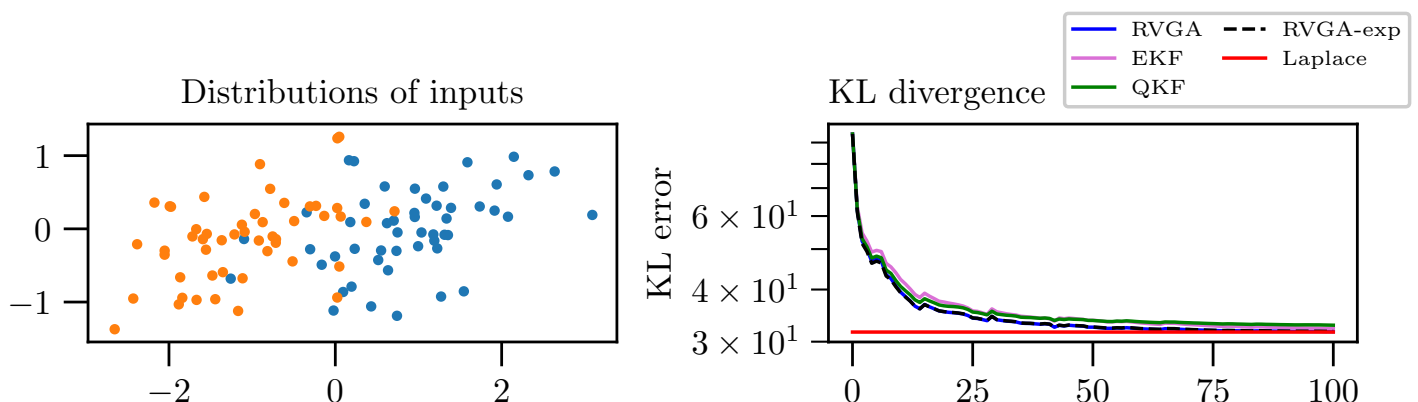

$\sigma_{0}=1,\left\|\mu_{0}\right\|=0.1, \mathrm{~N}=100, \mathrm{~d}=2, \mathrm{~s}=2.0, \mathrm{c}=2$
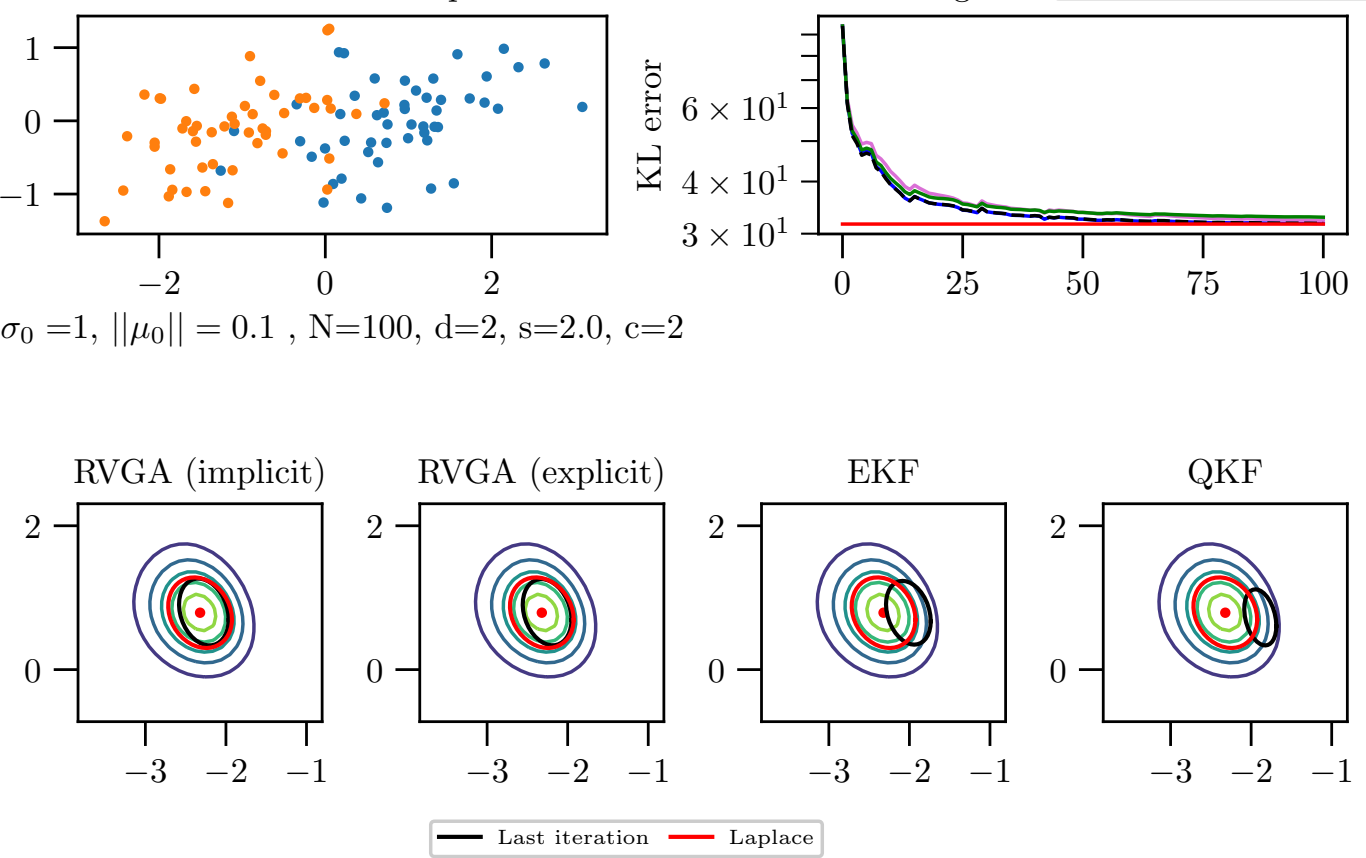

Figure 3: Case with $\sigma_{0}=1$ and $\left\|\mu_{0}\right\|=0.1$. Upper row: 2D synthetic dataset (left) and KL divergence. Lower row: Confidence ellipsoids for the R-VGA (left) vs EKF (middle) and QKF (right) at final time. The Batch Laplace covariance ellipsoid is shown in red and the contour lines of the true posterior are also displayed. Note that in this plot and the following the KL divergence is not normalized (as the normalizing constant is not known explicitly), so the upper plots exactly reflect how the algorithms perform relatively to each other but do not provide an indication of their absolute performance.

is the partition function and $\tilde{p}\left(\theta \mid Y_{N}\right)=\Pi_{1}^{N} p\left(y_{t} \mid \theta\right) p_{0}(\theta)$ is the unnormalized posterior. The divergence to the posterior of any Gaussian density $q$ with covariance $Q$ may be re-written:

$$
\begin{aligned}
& K L\left(q(\theta) \| p\left(\theta \mid Y_{N}\right)\right)=\int q(\theta) \log \frac{q(\theta)}{p\left(\theta \mid Y_{N}\right)}=\int q(\theta) \log \frac{q(\theta)}{\tilde{p}\left(\theta \mid Y_{N}\right)}+\log Z_{p} \\
& =-\int q(\theta) \log \tilde{p}\left(\theta \mid Y_{N}\right)-\frac{1}{2} \log |Q|-\frac{d}{2}(1+\log (2 \pi))+\log Z_{p} .
\end{aligned}
$$

To plot the evolution of the KL, we propose to draw $M$ independent samples $\theta_{k} \sim q$, leading to the Monte-Carlo approximation:

$$
K L \approx-\frac{1}{M} \sum_{k=1}^{M} \log \tilde{p}\left(\theta_{k} \mid Y_{N}\right)-\frac{1}{2} \log |Q|-\frac{d}{2}(1+\log (2 \pi))+\log Z_{p} .
$$

The normalization factor $Z_{p}$ can take very low values in high dimension and may prove difficult to estimate. As we aim at comparing the algorithms, we may arbitrarily set it to 1 (hence the plotted KL can take higher values than the true $\mathrm{KL}$ ). As it affects all $\mathrm{KL}$ evaluations in the same way, letting $Z_{p}=1$ allows for relative comparisons. 

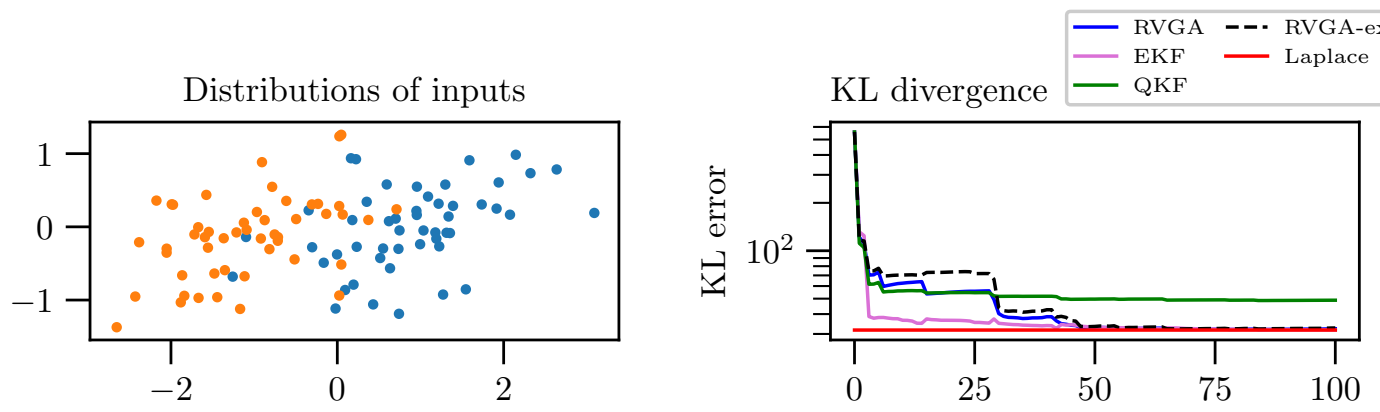

$\sigma_{0}=10,\left\|\mu_{0}\right\|=1.0, \mathrm{~N}=100, \mathrm{~d}=2, \mathrm{~s}=2.0, \mathrm{c}=2$
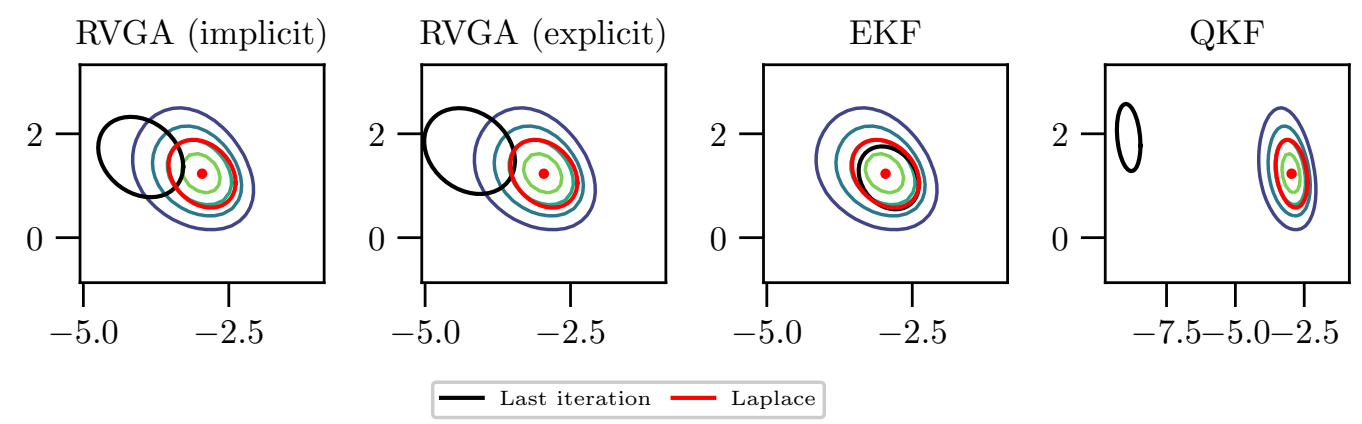

Figure 4: Same as Figure 3 in the case where $\sigma_{0}=10$ and $\left\|\mu_{0}\right\|=1$.
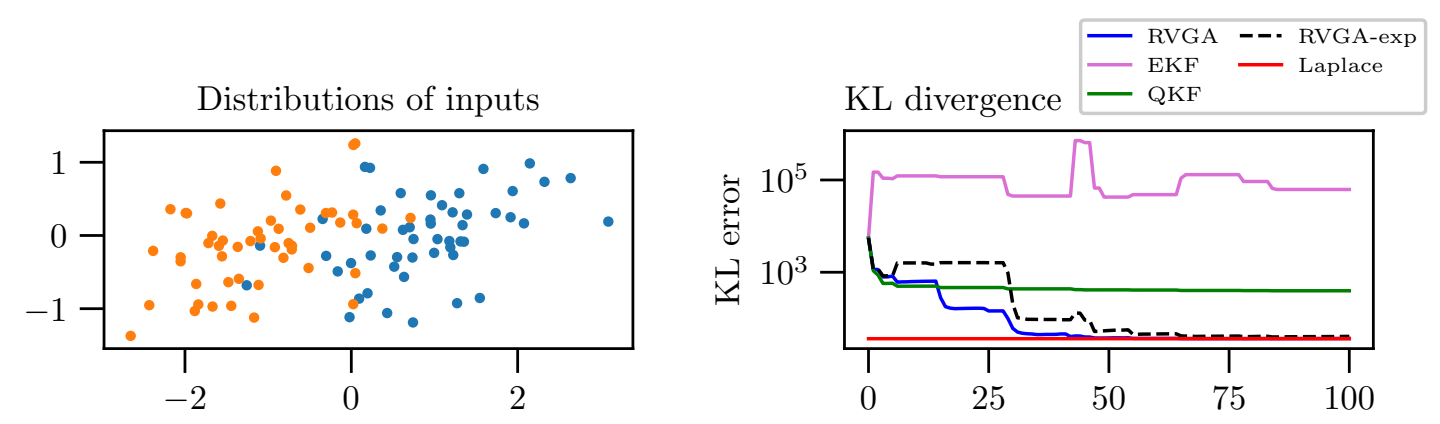

$\sigma_{0}=100,\left\|\mu_{0}\right\|=10.0, \mathrm{~N}=100, \mathrm{~d}=2, \mathrm{~s}=2.0, \mathrm{c}=2$
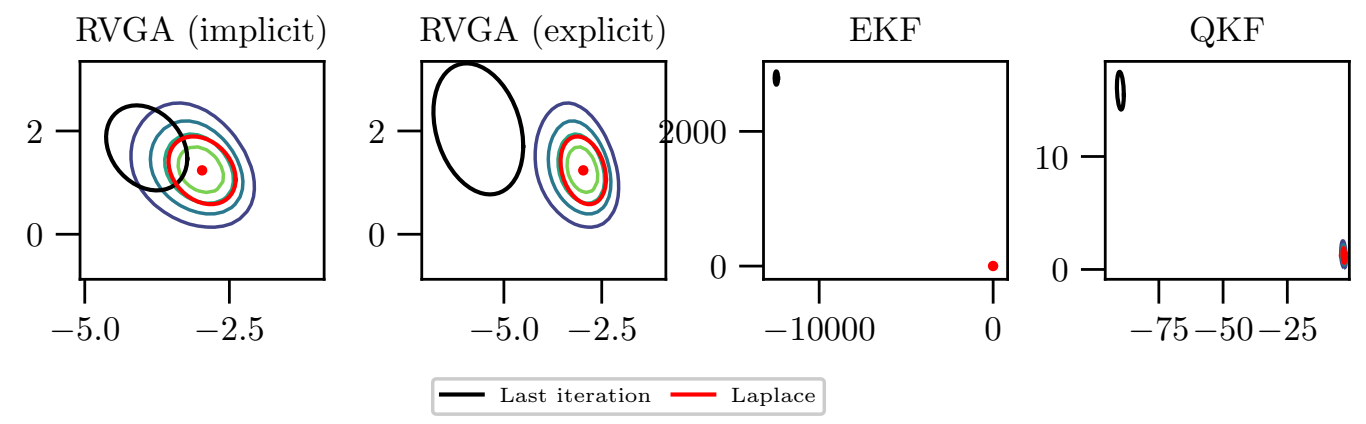

Figure 5: Same as Figure 3 in the case where $\sigma_{0}=100$ and $\left\|\mu_{0}\right\|=10$. 

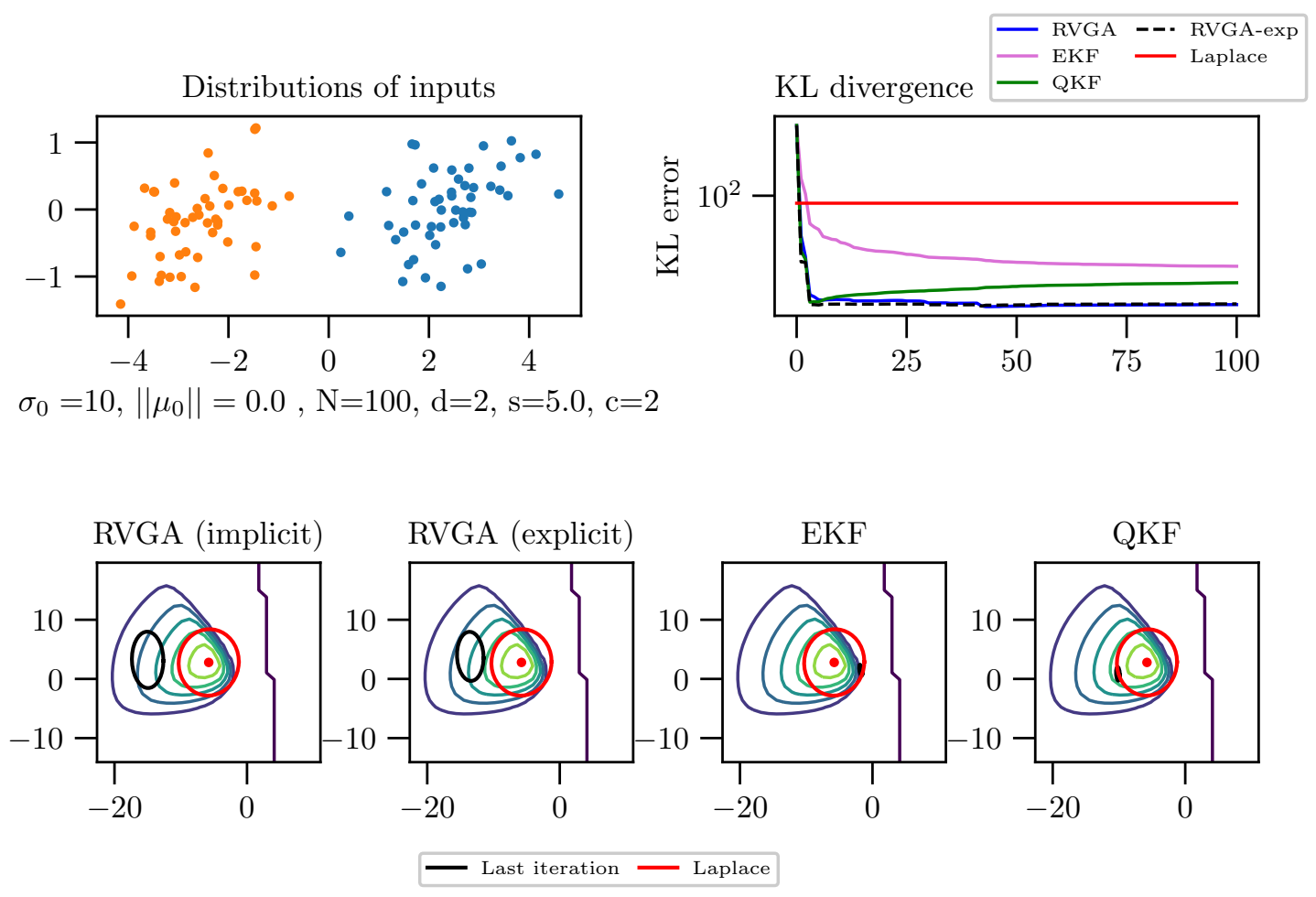

Figure 6: Same as Figure 3 in the harder case where the inputs distribution is ill-conditioned with $\mu_{0}$ set to 0 . The plots illustrate that the Gaussian Laplace approximation may be beaten in terms of KL divergence when the true posterior is asymmetric.

\subsection{Two-dimensional results}

We begin with a two-dimensional parameter $\theta$, that is, $d=2$ and a sample size $N$ of a few hundreds. This allows for plotting the confidence ellipsoids. In dimension two we use a grid to compute the true posterior and consider the batch Laplace approximation (see, e.g., Bishop [2006]) as a baseline, even if it can be biased for asymmetric sharp posteriors.

The form of the posterior is sharp when the data are separable, that is when the parameter $s$ reflecting separability is high. The posterior is sharper also when the prior has low confidence or equivalently when the problem is not regularized, that is $\sigma_{0}$ is large. In this case the maximum likelihood coincides with the maximum of the posterior. On the contrary, when $\sigma_{0}$ is very low, it dominates the observations, and the problem becomes approximately linear. The parameter $\mu_{0}$ often represents our initial guess, and might be set to a wrong value by the user. The more misaligned with the separating hyperplane the more challenging for the algorithms. The extended Kalman filter proves very sensitive to this initial guess, whereas the R-VGA is much less sensitive to large $\mu_{0}$ or $\sigma_{0}$.

We compare the R-VGA with the EKF and the variational approach based on a quadratic upper bound (QKF) detailed in the previous section. The ellipsoids of the covariance matrix at final time and the (unnormalized) KL divergence are shown for different values of the prior parameters $\mu_{0}$ and $\sigma_{0}$ (Figures 3 to 5) where we have supposed here $\mu_{0}$ to be proportional to the unitary vector of $\mathbb{R}^{d}$. Note the seemingly contradictory fact that the KL divergence may seem to take very small values even when the corresponding approximation is far from the true posterior is because we are using an unnormalized KL divergence. This allows for relative comparisons between algorithms but does not provide an indication of absolute performance. 
Finally, regarding comparison metrics, note that when the loss is sharper, the covariance given by the Laplace approximation spills out the true posterior to regions of very low probability whereas the R-VGA avoids them. As a consequence Laplace approximation is strongly penalized with the left-sided KL whereas it may perform better for a right-sided KL or the Jenson-Shannon divergence. Unfortunately, those metrics are not easily computable for the considered experiment.

A harder case where the data are ill-conditioned is shown in Figure 6. More examples are shown in Appendix 9.6. We see that (exact) R-VGA, which is based on successive optimal Gaussian approximations, generally outperforms the EKF and QKF. The R-VGA explicit scheme yields results that are close to the R-VGA implicit scheme's but tend to degrade for large $\sigma_{0}$.

We note the EKF performs quite well on some experiments, and might even beat R-VGA sometimes, see Figure 4, but is outperformed most of the time and may even prove unreliable as shown in Figure 5. It has long been known indeed that the behavior of the EKF is problem-dependent. It can perform very well for some problems, for instance the EKF has been the standard algorithm for navigation and guidance for decades. It is not completely surprising it may beat R-VGA, as both algorithms are based on successive approximations (even if the R-VGA is optimal at each step, its optimality concerns an approximated problem). Indeed, a caveat that shall be borne in mind is that all the online recursive algorithms treat data one by one and then discard the data. This makes their use advantageous for large sample size, but inherently approximate.

However, the EKF is known to be prone to divergence in some cases, and no genuine guarantees exist about its behavior in general, see, e.g., Barrau and Bonnabel [2018] and discussion therein. On the other hand, R-VGA is locally optimal at each step, since it provides the closest Gaussian to a relevant approximated posterior. It is thus more robust, in that the approximation it makes is controlled and as a result cannot wholly diverge. The EKF is less robust as it is based on a first-order approximation that may be erroneous for nonlinear problems. In the case of static state (as in the present paper), divergence has been observed due to the EKF working with linear approximations, and hence decreasing too rapidly its gains for the nonlinearities to be overcome [Barrau and Bonnabel, 2016]. This leads the engineers to spuriously inflate the process noise covariance matrix - a technique known as robust tuning - making the algorithm address a different Bayesian inference problem than the one at hand.

\subsection{Higher dimension results}

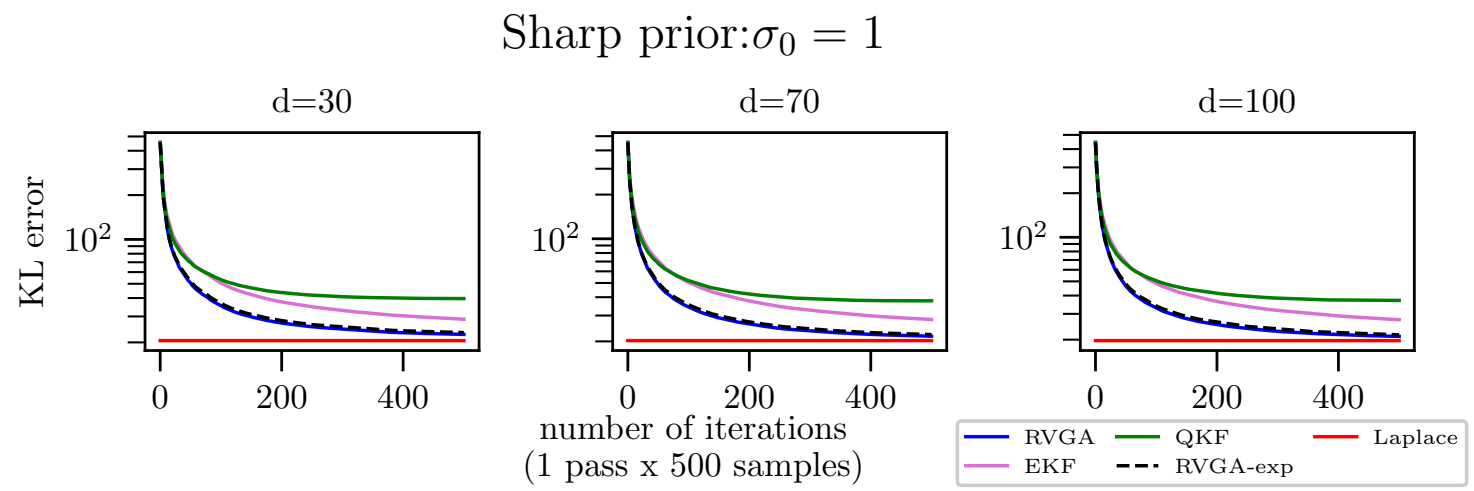

Figure 7: $\sigma_{0}=1$ and $\mu_{0}=0$. Evolution of the convergence for $d=30,70$ and 100 for $N=500$ with an "isotropic" covariance.

In higher dimension we study the sensitivity to the dimension $d$ of the parameter for a sharp prior encoded in $\sigma_{0}$ (Figure 7), and a flat prior (Figure 8). We see the R-VGA outperforms its Kalman-based counterparts. When the prior is weighty the discrepancies between algorithms look more moderate than when $\sigma_{0}$ is large, 


\section{Flat prior: $\sigma_{0}=30$}
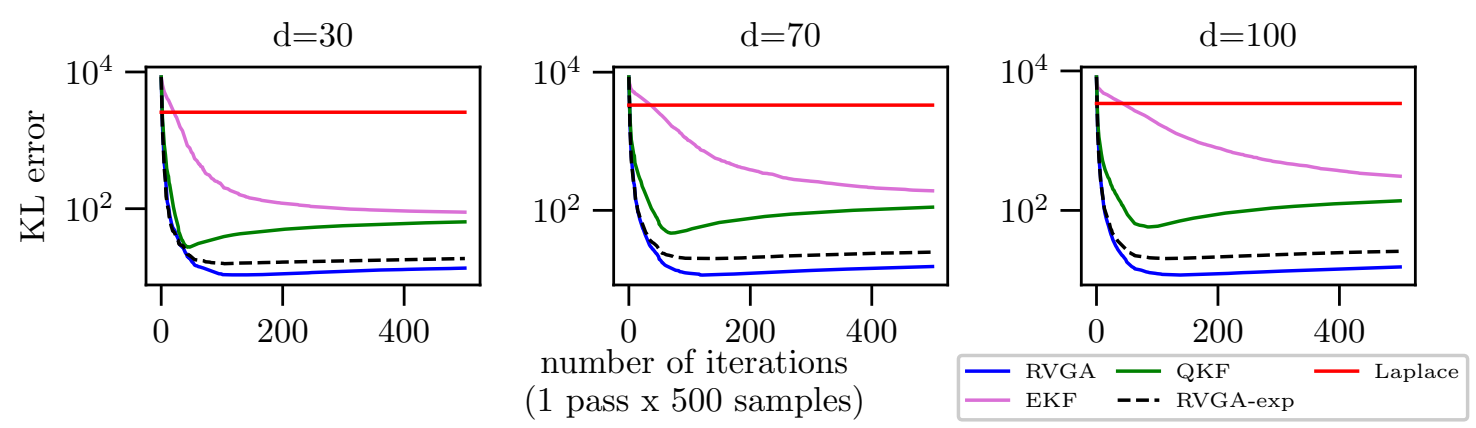

Figure 8: $\sigma_{0}=30$ and $\mu_{0}=0$. Evolution of the convergence for $d=30,70$ and 100 for $N=500$ and an "isotropic" covariance.
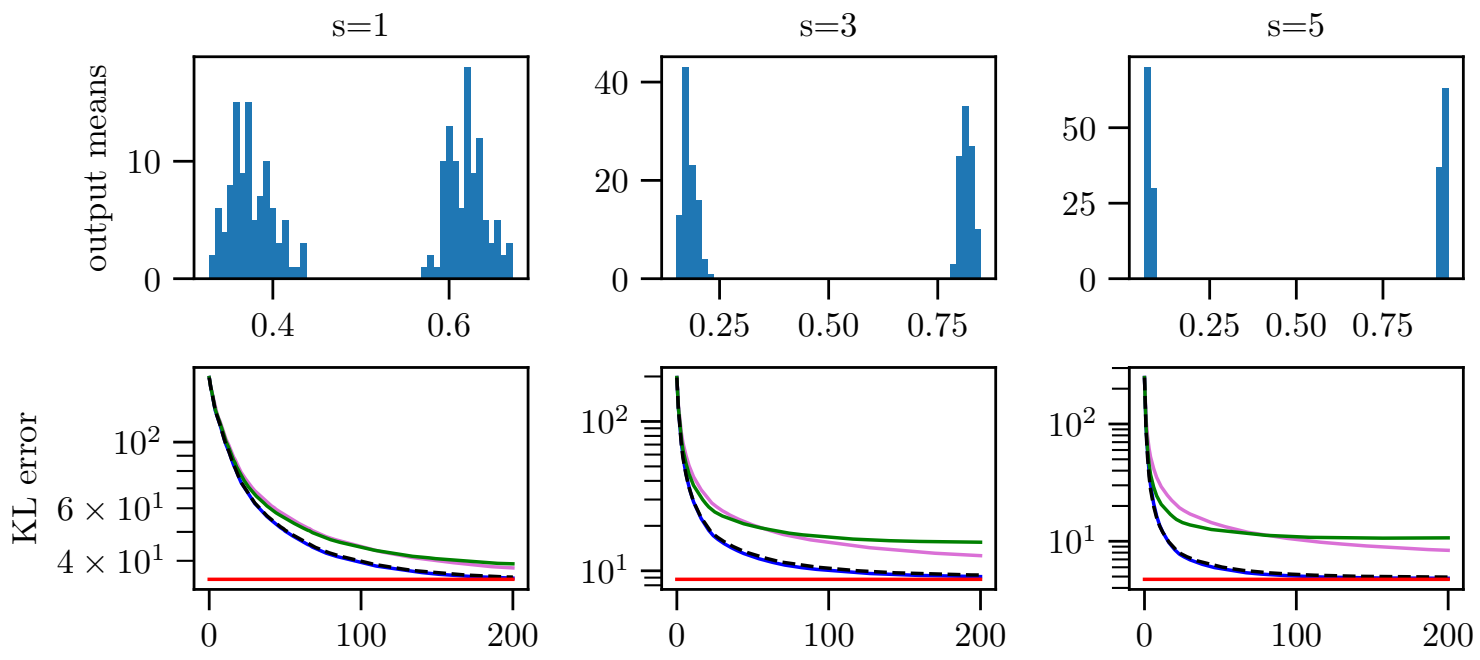

number of iterations

(1 pass x 200 samples)

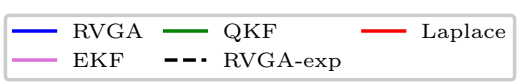

Figure 9: $\sigma_{0}=10$ and $\mu_{0}=0$. Evolution of the convergence with the separability of the inputs means $s$ for $s=1,3$ and 5 . We have generated $N=200$ inputs in dimension $d=100$ and an "isotropic" covariance.

in which case differences are stark. We assess also the sensitivity to the separability of the dataset $s$ (Figures 9 and 10). This parameter drives the sharpness of the posterior. We see that R-VGA outperforms the other Kalman variants and is even susceptible to beat the batch Laplace approximation because it avoids the region of near null probabilities whereas the Laplace method does not.

Interestingly, the R-VGA explicit scheme runs a close second in most experiments, especially if we compare final estimates. Notably, it seems more reliable than the EKF. This is a reassuring feature that confirms the intuition that replacing parameters of the distribution at current step with those at previous step as is done in the explicit scheme does not come at a great cost in terms of performance. However, by using 

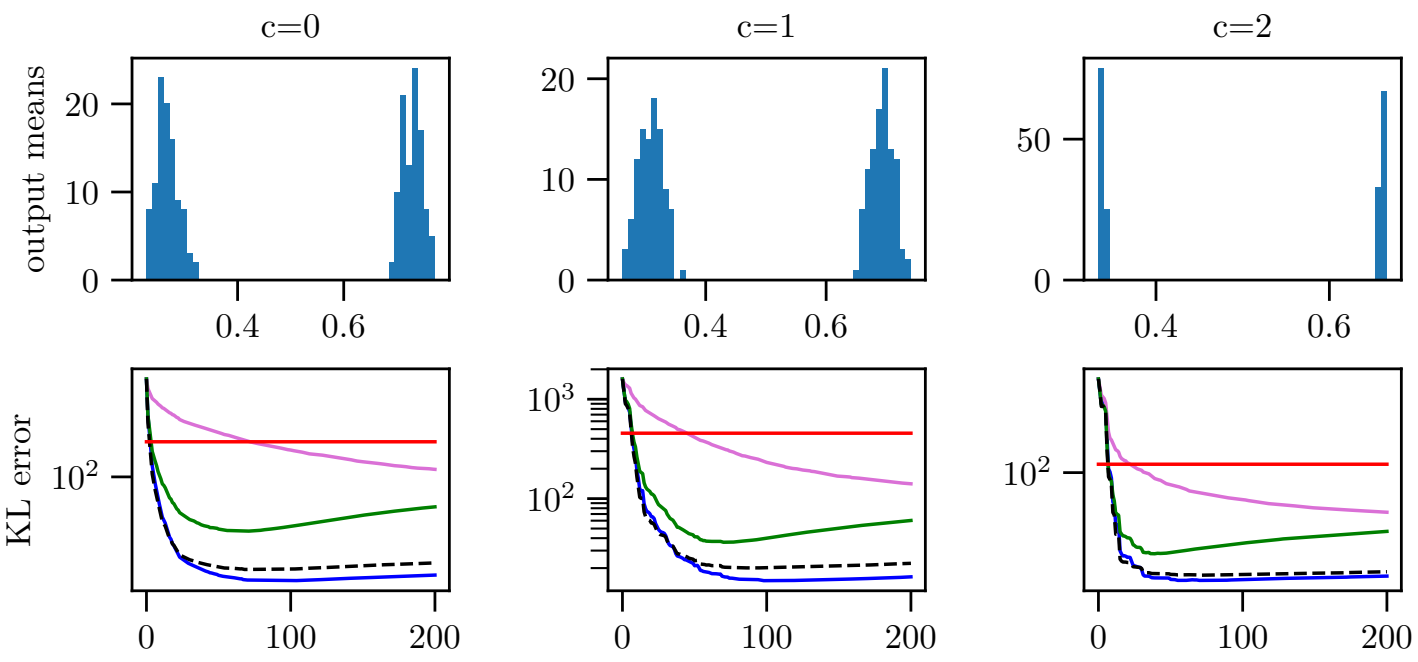

number of iterations

(1 pass x 200 samples)

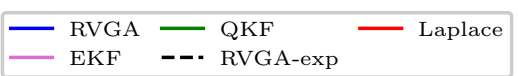

Figure 10: $\sigma_{0}=10$ and $\mu_{0}=0$. More challenging case of an ill-conditioned covariance matrix for the inputs. The separation of the data depends here to the geometry of the problem. We have generated $N=200$ inputs in dimension $d=100$.

the explicit scheme we lose the optimality guarantees that the implicit scheme possesses, unless the problem is linear and Gaussian, or the model linearized, in which case we recall that both algorithms are identical (see Theorems 2 and 3 respectively).

Sources: The sources of the code are available on Github on the following repository: https://github. $\mathrm{com} / \mathrm{marc}-\mathrm{h}$-lambert/Kalman4Classification.

\section{Conclusion}

We have shown how a recursive version of the variational Gaussian approximation leads to a new family of implicit optimization algorithms which generalize the extended Kalman filter and the natural gradient in the exponential family case. This recursive version allows for sequential processing of the data which is suited to large sample size as is characteristic of big data. Moreover, owing to the nature of the considered problem, that is, infer a posterior distribution, only one pass through the data is required. The algorithm performs a sequence of optimal Gaussian approximations, which comes at the price of an implicit update scheme. For linear regression R-VGA is optimal and shown to coincide with the Kalman filter. For the logistic regression problem, a computable form was obtained. In this case, the algorithm proves to scale relatively with the parameter dimension $d$, with an overall computation $\operatorname{cost} O\left(d^{2} N\right)$. While having similar computational cost, R-VGA beats the extended Kalman filter or equivalently the online natural gradient, see Ollivier [2018], as well as the variational approach with quadratic upper bound we have proposed, inspired from Jaakkola and Jordan [1997]. When the posterior has a tall spike, though, we have observed the method fails to recover 
the true posterior, which is to be expected as it is based on a series of Gaussian approximations. However, this method does not require any tuning, and could be used to initialize more precise and computationally demanding MCMC methods to speed up their convergence. In the future, we plan to address issues related to high dimensional problems, and hence to devise a low-rank version of R-VGA that scales linearly with $d$. Another route would be to attempt to adapt the proposed method to models involving latent variables, like probabilistic PCA.

\section{Acknowledgements}

This work was funded in part by the French government under management of Agence Nationale de la Recherche as part of the "Investissements d'avenir" program, reference ANR-19-P3IA-0001(PRAIRIE 3IA Institute). We also acknowledge support from the French Defence procurement agency (DGA) and from the European Research Council (grant SEQUOIA 724063). The authors would like to thank Eric Moulines and Jean-Pierre Nouaille as well as Hadi Daneshmand for fruitful discussions related to this work.

\section{References}

D. Barber and Christopher Bishop. Ensemble learning in Bayesian neural networks. In Generalization in Neural Networks and Machine Learning, pages 215-237, 1998.

Timothy D Barfoot, James R Forbes, and David J Yoon. Exactly sparse gaussian variational inference with application to derivative-free batch nonlinear state estimation. The International Journal of Robotics Research, 39(13):1473-1502, 2020. doi: 10.1177/0278364920937608.

Axel Barrau and Silvere Bonnabel. Navigating with highly precise odometry and noisy GPS: a case study. IFAC-PapersOnLine, 49(18):618-623, 2016.

Axel Barrau and Silvere Bonnabel. Invariant Kalman filtering. Annual Review of Control, Robotics, and Autonomous Systems, 1:237-257, 2018.

Dimitri P. Bertsekas. Incremental least squares methods and the extended Kalman filter. SIAM Journal on Optimization, 6(3):807-822, 1996.

G. J. Bierman. Measurement updating using the U-D factorization. In Conference on Decision and Control including the Symposium on Adaptive Processes, pages 337-346, 1975.

Christopher M. Bishop. Pattern Recognition and Machine Learning. springer, 2006.

Tamara Broderick, Nicholas Boyd, Andre Wibisono, Ashia C Wilson, and Michael I Jordan. Streaming variational Bayes. In Advances in Neural Information Processing Systems, volume 26, 2013.

Kamélia Daudel, Randal Douc, and François Portier. Infinite-dimensional gradient-based descent for alphadivergence minimisation. The Annals of Statistics, 49:2250 - 2270, 2021.

Jean Daunizeau. Semi-analytical approximations to statistical moments of sigmoid and softmax mappings of normal variables. arXiv preprint arXiv:1703.00091, 2017.

Mohammad Emtiyaz Khan, Zuozhu Liu, Voot Tangkaratt, and Yarin Gal. Vprop: Variational inference using RMSprop. arXiv, pages arXiv-1712, 2017.

Yarin Gal. Uncertainty in deep learning. University of Cambridge, 2016. 
Elad Hazan, Amit Agarwal, and Satyen Kale. Logarithmic regret algorithms for online convex optimization. Machine Learning, 69:169-192, 2007.

Yumei Hu, Xuezhi Wang, Hua Lan, Zengfu Wang, Bill Moran, and Quan Pan. An iterative nonlinear filter using variational Bayesian optimization. Sensors, 18(12), 2018. ISSN 1424-8220.

Tommi Jaakkola and Michael Jordan. A variational approach to Bayesian logistic regression models and their extensions. In Sixth International Workshop on Artificial Intelligence and Statistics, volume 82, 1997.

Rémi Jézéquel, Pierre Gaillard, and Alessandro Rudi. Efficient improper learning for online logistic regression. arXiv preprint arXiv:2003.08109, 2020.

Mohammad Emtiyaz Khan and Wu Lin. Conjugate-computation variational inference: Converting variational inference in non-conjugate models to inferences in conjugate models. In AISTATS, pages 878-887, 2017.

Mohammad Emtiyaz Khan and Didrik Nielsen. Fast yet simple natural-gradient descent for variational inference in complex models. arXiv-1807.04489, 2018.

Mohammad Emtiyaz Khan, Didrik Nielsen, Voot Tangkaratt, Wu Lin, Yarin Gal, and Akash Srivastava. Fast and scalable bayesian deep learning by weight-perturbation in Adam. arXiv preprint arXiv:1806.04854, 2018.

Frederik Kunstner, Philipp Hennig, and Lukas Balles. Limitations of the empirical Fisher approximation for Natural gradient descent. In Advances in Neural Information Processing Systems, pages 4156-4167, 2019.

Wu Lin, Mohammad Emtiyaz Khan, and Mark Schmidt. Fast and simple natural-gradient variational inference with mixture of exponential-family approximations. arXiv preprint arXiv:1906.02914, 2019a.

Wu Lin, Mohammad Emtiyaz Khan, and Mark Schmidt. Stein's lemma for the reparameterization trick with exponential family mixtures. arXiv preprint arXiv:1910.13398, $2019 \mathrm{~b}$.

James Martens. New insights and perspectives on the natural gradient method. Journal of Machine Learning Research, 21(146):1-76, 2020.

James McInerney, Rajesh Ranganath, and David Blei. The population posterior and bayesian modeling on streams. In Advances in Neural Information Processing Systems, volume 28, 2015.

Yurii Nesterov and Vladimir Spokoiny. Random gradient-free minimization of convex functions. Foundations of Computational Mathematics, 17:527-566, 2017.

Yann Ollivier. Online Natural gradient as a Kalman filter. Electronic Journal of Statistics, 12:2930-2961, 2018.

M. J. D. Powell. On nonlinear optimization since 1959. In The Birth of Numerical Analysis, pages 141-160. 2010.

Rajesh Ranganath, Sean Gerrish, and David Blei. Black box variational inference. In Artificial intelligence and statistics, pages 814-822. PMLR, 2014.

Herbert Robbins and Sutton Monro. A stochastic approximation method. The annals of mathematical statistics, pages 400-407, 1951.

Masa-Aki Sato. Online model selection based on the variational bayes. Neural Comput., 13(7):1649-1681, July 2001. ISSN 0899-7667. doi: 10.1162/089976601750265045. 
Mark Schmidt, Nicolas Le Roux, and Francis Bach. Minimizing finite sums with the stochastic average gradient. Mathematical Programming, 162(1-2):83-112, 2017.

VÁclav Smidl and Anthony Quinn. Variational bayesian filtering. IEEE Transactions on Signal Processing, 56(10):5020-5030, 2008. doi: 10.1109/TSP.2008.928969.

Peter Sykacek and Stephen J Roberts. Adaptive classification by variational kalman filtering. In Advances in Neural Information Processing Systems, volume 15. MIT Press, 2003.

Michalis Titsias and Miguel Lázaro-Gredilla. Doubly stochastic variational Bayes for non-conjugate inference. In International conference on machine learning, pages 1971-1979, 2014.

Martin J. Wainwright and Michael I. Jordan. Graphical models, exponential families, and variational inference. Foundations and Trends ${ }^{\circledR}$ in Machine Learning, 1(1-2):1-305, 2008. ISSN 1935-8237. doi: $10.1561 / 2200000001$.

Steve Waterhouse, David Mackay, and Tony Robinson. Bayesian Methods for Mixtures of Experts. MIT Press, 1996.

Florian Wenzel, Théo Galy-Fajou, Christan Donner, Marius Kloft, and Manfred Opper. Efficient gaussian process classification using Pòlya-Gamma data augmentation. In Proceedings of the AAAI Conference on Artificial Intelligence, volume 33, pages 5417-5424, 2019.

\section{Appendix}

\subsection{Generalization of Theorem 1}

Theorem 1 can be generalized to any target distribution belonging to an exponential family of natural parameter $\eta$ as follows:

Theorem 4. Considering an exponential family $q_{\eta}$ of natural parameter $\eta$, mean parameter $m$ and $a$ strictly convex $\log$ partition function $F$ such that $\left.q_{\eta}(\theta)=h(\theta) \exp (<\eta, \theta\rangle-F(\eta)\right)$, the solution to the recursive variational approximation problem between a target distribution $q_{\eta}$ and the one-sample posterior $p\left(\theta \mid y_{t}\right) \propto p\left(y_{t} \mid \theta\right) q_{\eta_{t-1}}(\theta):$

$$
\underset{\eta_{t}}{\arg \min } K L\left(q_{\eta_{t}}(\boldsymbol{\theta}) \mid p\left(\boldsymbol{\theta} \mid y_{t}\right)\right)
$$

must satisfy the following implicit fixed point equation on the natural parameter:

$$
\begin{aligned}
\eta_{t} & =\eta_{t-1}+\left(\nabla^{2} F\left(\eta_{t}\right)\right)^{-1} \nabla_{\eta_{t}} \mathbf{E}_{q_{\eta_{t}}}\left[\log p\left(y_{t} \mid \theta\right)\right] \\
& =\eta_{t-1}+\nabla_{m_{t}} \mathbf{E}_{q_{m_{t}}}\left[\log p\left(y_{t} \mid \boldsymbol{\theta}\right)\right] .
\end{aligned}
$$

If $q_{\eta}$ is a Gaussian distribution, this fixed point equation is equivalent to the R-VGA updates given in Theorem 1.

This theorem extends the results found in Khan and Lin [2017] and Khan and Nielsen [2018] to the recursive variational inference framework. Here, the implicit updates come directly from the critical point of our recursive form (95) and not from the application of an explicit natural gradient. 
Proof. The recursive variational approximation for the target distribution $q_{\eta}$ of log-partition function $F$ may be re-written as:

$$
\begin{aligned}
K L\left(q_{\eta_{t}}(\boldsymbol{\theta}) \mid p\left(\boldsymbol{\theta} \mid y_{t}\right)\right) & =\mathbf{E}_{q_{\eta_{t}}}\left[-\log p\left(y_{t} \mid \boldsymbol{\theta}\right)\right]+K L\left(q_{\eta_{t}}(\boldsymbol{\theta}) \mid q_{\eta_{t-1}}(\boldsymbol{\theta})\right)+c \\
& =\mathbf{E}_{q_{\eta_{t}}}\left[-\log p\left(y_{t} \mid \boldsymbol{\theta}\right)\right]+B_{F}\left(\eta_{t-1}, \eta_{t}\right)+c,
\end{aligned}
$$

where $c$ is a normalization constant and $B_{F}$ is the Bregman divergence associated to the strictly convex $\log$ partition function $F$ :

$$
B_{F}\left(\eta_{1}, \eta_{2}\right)=F\left(\eta_{1}\right)-F\left(\eta_{2}\right)-<\eta_{1}-\eta_{2}, \nabla F\left(\eta_{2}\right)>
$$

The derivative of the variational divergence (99) with respect to the natural parameter $\eta_{t}$ gives:

$$
\begin{aligned}
& \nabla_{\eta_{t}} \mathbf{E}_{q_{\eta_{t}}}\left[-\log p\left(y_{t} \mid \theta\right)\right]+\left(\eta_{t}-\eta_{t-1}\right) \nabla^{2} F\left(\eta_{t}\right)=0 \\
& \Longleftrightarrow \\
& \eta_{t}=\eta_{t-1}+\left(\nabla^{2} F\left(\eta_{t}\right)\right)^{-1} \nabla_{\eta_{t}} \mathbf{E}_{q_{\eta_{t}}}\left[\log p\left(y_{t} \mid \theta\right)\right] \\
&=\eta_{t-1}+\nabla_{m_{t}} \mathbf{E}_{q_{m_{t}}}\left[\log p\left(y_{t} \mid \boldsymbol{\theta}\right)\right],
\end{aligned}
$$

which yields the desired result. The last equality comes from the properties of exponential families with a strictly convex $\log$-partition function $F$ : the mean parameter $m$ is the Legendre dual of the natural parameter $\eta$, ie $<\eta, m>=F(\eta)+F^{*}(m)$ and $\nabla^{2} F(\eta)=\frac{\partial m}{\partial \eta}$.

Finally, if we apply this equation to the natural parameter for a Gaussian distribution $\eta=\left(\begin{array}{c}P^{-1} \mu \\ -\frac{1}{2} P^{-1}\end{array}\right)$, we retrieve the R-VGA updates. Indeed, to derive the R-VGA from the implicit version of the natural gradient we use the chain rule to relate the mean parameters to the natural parameters as proposed by Khan \& al, in the context of batch variational approximation, in Khan et al. [2018] (appendix $C$ ). In the Gaussian case the mean is $m=\left(\begin{array}{c}m_{1}=\mu \\ m_{2}=P+\mu \mu^{T}\end{array}\right)$ and the natural parameter is $\eta=\left(\begin{array}{c}\eta_{1}=P^{-1} \mu \\ \eta_{2}=-\frac{1}{2} P^{-1}\end{array}\right)$. The gradient with respect to the mean parameters $m_{1}, m_{2}$ can be expressed as the gradient with respect to the sources parameters $\mu, P$ using the chain rule:

$$
\begin{aligned}
\frac{\partial f}{\partial m_{1}} & =\frac{\partial f}{\partial \mu} \frac{\partial \mu}{\partial m_{1}}+\frac{\partial f}{\partial P} \frac{\partial P}{\partial m_{1}}=\frac{\partial f}{\partial \mu}-2 \frac{\partial f}{\partial P} \mu \\
\frac{\partial f}{\partial m_{2}} & =\frac{\partial f}{\partial \mu} \frac{\partial \mu}{\partial m_{2}}+\frac{\partial f}{\partial P} \frac{\partial P}{\partial m_{2}}=\frac{\partial f}{\partial P}
\end{aligned}
$$

The updates

$$
\eta_{t}=\eta_{t-1}+\nabla_{m_{t}} \mathbf{E}_{q_{\eta_{t}}}\left[\log p\left(y_{t} \mid \boldsymbol{\theta}\right)\right]
$$

become:

$$
\begin{aligned}
& \left(\begin{array}{c}
P_{t}^{-1} \mu_{t} \\
-\frac{1}{2} P_{t}^{-1}
\end{array}\right)=\left(\begin{array}{c}
P_{t-1}^{-1} \mu_{t-1} \\
-\frac{1}{2} P_{t-1}^{-1}
\end{array}\right)+\left(\begin{array}{c}
\frac{\partial}{\partial \mu_{t}} \mathbf{E}_{q \eta_{t}}\left[\log p\left(y_{t} \mid \boldsymbol{\theta}\right)\right]-2 \frac{\partial}{\partial P_{t}} \mathbf{E}_{q_{\eta_{t}}}\left[\log p\left(y_{t} \mid \boldsymbol{\theta}\right)\right] \mu_{t} \\
\frac{\partial}{\partial P_{t}} \mathbf{E}_{q_{\eta_{t}}}\left[\log p\left(y_{t} \mid \boldsymbol{\theta}\right)\right]
\end{array}\right) \\
& \left(\begin{array}{c}
\left(P_{t}^{-1}+2 \frac{\partial}{\partial P_{t}} \mathbf{E}_{q \eta_{t}}\left[\log p\left(y_{t} \mid \boldsymbol{\theta}\right)\right]\right) \mu_{t} \\
P_{t}^{-1}
\end{array}\right)=\left(\begin{array}{c}
P_{t-1}^{-1} \mu_{t-1} \\
P_{t-1}^{-1}
\end{array}\right)+\left(\begin{array}{c}
\frac{\partial}{\partial \mu_{t}} \mathbf{E}_{q_{\eta_{t}}}\left[\log p\left(y_{t} \mid \boldsymbol{\theta}\right)\right] \\
-2 \frac{\partial}{\partial P_{t}} \mathbf{E}_{q_{\eta_{t}}}\left[\log p\left(y_{t} \mid \boldsymbol{\theta}\right)\right]
\end{array}\right) \\
& \left(\begin{array}{c}
\mu_{t} \\
P_{t}^{-1}
\end{array}\right)=\left(\begin{array}{c}
\mu_{t-1} \\
P_{t-1}^{-1}
\end{array}\right)+\left(\begin{array}{c}
P_{t-1} \frac{\partial}{\partial \mu_{t}} \mathbf{E}_{q_{\eta_{t}}}\left[\log p\left(y_{t} \mid \boldsymbol{\theta}\right)\right] \\
-2 \frac{\partial}{\partial P_{t}} \mathbf{E}_{q_{\eta_{t}}}\left[\log p\left(y_{t} \mid \boldsymbol{\theta}\right)\right]
\end{array}\right),
\end{aligned}
$$

which are the R-VGA equations indeed. 


\subsection{Proof of Theorem 2}

Proof. In the case where $p$ is a multivariate Gaussian distribution $p\left(y_{t} \mid \theta\right) \sim \mathscr{N}\left(y_{t} \mid H_{t} \theta, R_{t}\right)$, with $H_{t}$ is the observation matrix, we have the relation:

$$
\begin{aligned}
\log p\left(y_{t} \mid \theta\right) & =-\frac{1}{2}\left(y_{t}-H_{t} \theta\right)^{T} R_{t}^{-1}\left(y_{t}-H_{t} \theta\right)+C=-\ell_{t}(\theta) \\
\nabla_{\mu_{t}} \mathbf{E}_{q_{t}}\left[\log p\left(y_{t} \mid \theta\right)\right] & =H_{t}^{T} R_{t}^{-1}\left(y_{t}-H_{t} \mu_{t}\right)=-\nabla \ell_{t}(\theta) \\
-2 \nabla_{P_{t}} \mathbf{E}_{q_{t}}\left[\log p\left(y_{t} \mid \theta\right)\right] & =H_{t}^{T} R_{t}^{-1} H_{t}=\nabla^{2} \ell_{t}(\theta),
\end{aligned}
$$

this last relation gives directly the second R-VGA update equation, rewriting (2) as:

$$
P_{t}^{-1}=P_{t-1}^{-1}+H_{t}^{T} R_{t}^{-1} H_{t}
$$

which is the information update equation. We then rewrite the first R-VGA update equation (1) as:

$$
\begin{aligned}
& \mu_{t}=\mu_{t-1}+P_{t-1} H_{t}^{T} R_{t}^{-1}\left(y_{t}-H_{t} \mu_{t}\right) \\
& \quad \Longleftrightarrow \mu_{t}\left(\mathbf{I}_{d}+P_{t-1} H_{t}^{T} R_{t}^{-1} H_{t}\right)=\mu_{t-1}+P_{t-1} H_{t}^{T} R_{t}^{-1} y_{t} \\
& \quad \Longleftrightarrow \mu_{t}=\left(P_{t-1}^{-1}+H_{t}^{T} R_{t}^{-1} H_{t}\right)^{-1} P_{t-1}^{-1}\left(\mu_{t-1}+P_{t-1} H_{t}^{T} R_{t}^{-1} y_{t}\right) \\
& \Longleftrightarrow \mu_{t}=\mu_{t-1}+\left(P_{t-1}^{-1}+H_{t}^{T} R_{t}^{-1} H_{t}\right)^{-1}\left(-H_{t}^{T} R_{t}^{-1} H_{t} \mu_{t-1}+H_{t}^{T} R_{t}^{-1} y_{t}\right) \\
& \Longleftrightarrow \mu_{t}=\mu_{t-1}+P_{t} H_{t}^{T} R_{t}^{-1}\left(y_{t}-H_{t} \mu_{t-1}\right) .
\end{aligned}
$$

If we note the Kalman gain $K_{t}=P_{t} H_{t}^{T} R_{t}^{-1}$, we find the Kalman update equations for the state (42), indeed:

$$
K_{t}=P_{t} H_{t}^{T} R_{t}^{-1}=\left(P_{t-1}^{-1}+H_{t}^{T} R_{t}^{-1} H_{t}\right)^{-1} H_{t}^{T} R_{t}^{-1}=P_{t-1} H_{t}^{T}\left(R_{t}+H_{t} P_{t-1} H_{t}^{T}\right)^{-1},
$$

where we have used the matrix formula: $\left(A^{-1}+B^{T} C^{-1} B\right)^{-1} B^{T} C^{-1}=A B^{T}\left(B A B^{T}+C\right)^{-1}$. The Kalman update equation for the covariance matrix (42) is then deduced from (107) using the Woodbury formula:

$$
\begin{aligned}
P_{t}^{-1} & =P_{t-1}^{-1}+H_{t}^{T} R_{t}^{-1} H_{t} \\
\Longleftrightarrow P_{t} & =P_{t-1}-P_{t-1} H_{t}^{T}\left(R_{t}+H_{t} P_{t-1} H_{t}^{T}\right)^{-1} H_{t} P_{t-1} \\
& =\left(\mathbb{I}-K_{t} H_{t}\right) P_{t-1} .
\end{aligned}
$$

Equivalence to linear Kalman filter have thus been proved.

The equation (109) can be rewritten to find, combined with (107), the online Newton descent. Indeed, if we pose $Q_{t}=P_{t}^{-1}$ as the estimation of the Hessian matrix up to the iteration $t$ we find directly:

$$
\begin{aligned}
& \mu_{t}=\mu_{t-1}-Q_{t}^{-1} \nabla \ell_{t}(\theta) \\
& Q_{t}=Q_{t-1}+H_{t}^{T} R_{t}^{-1} H_{t} .
\end{aligned}
$$

This proves the equivalence to the online Newton descent. Now it is well known the Kalman filter is optimal for the least mean squares problem, let us reformulate the proof to better show the connection to stochastic optimization.

Let us recall the form of the least mean squares cost function for $t$ observations :

$$
\sum_{i=1}^{t} \ell_{i}(\theta)=\frac{1}{2} \theta^{T} Q_{t} \theta-v_{t}^{T} \theta+C
$$


We express now the optimal $\theta_{t}^{*}=Q_{t}^{-1} v_{t}$ at time $t$ in function of the optimal at time $t-1$ :

$$
\begin{aligned}
\theta_{t}^{*} & =Q_{t}^{-1} v_{t} \\
& =Q_{t}^{-1}\left(v_{t-1}+H_{t}^{T} R_{t}^{-1} y_{t}\right) \\
& =Q_{t}^{-1}\left(Q_{t-1} \theta_{t-1}^{*}+H_{t}^{T} R_{t}^{-1} y_{t}\right) \\
& =Q_{t}^{-1}\left(\left(Q_{t}-H_{t}^{T} R_{t}^{-1} H_{t}\right) \theta_{t-1}^{*}+H_{t}^{T} R_{t}^{-1} y_{t}\right) \\
& =\theta_{t-1}^{*}-Q_{t}^{-1} H_{t}^{T} R_{t}^{-1} H_{t} \theta_{t}^{*}+Q_{t}^{-1} H_{t}^{T} R_{t}^{-1} y_{t} \\
& =\theta_{t-1}^{*}+Q_{t}^{-1} H_{t}^{T} R_{t}^{-1}\left(y_{t}-H_{t} \theta_{t-1}^{*}\right) \\
& =\theta_{t-1}^{*}-Q_{t}^{-1} \nabla \ell_{t}(\theta) \\
& =\theta_{t-1}^{*}+K_{t}\left(y_{t}-H_{t} \theta_{t-1}^{*}\right) .
\end{aligned}
$$

The two last equations show that the recursive least mean squares estimate is found by both online Newton and the linear Kalman filter.

\subsection{Proof of Theorem 3}

Proof. The proof is quite similar to the proof in the linear case. The update equation (3) can be rewritten using the same manipulations as in the linear case:

$$
\begin{aligned}
\mu_{t} & =\mu_{t-1}-P_{t-1} \mathbf{E}_{q_{t}}\left[\nabla_{\theta} \ell_{t}(\theta)\right] \\
& =\mu_{t-1}+P_{t-1} \mathbf{E}_{q_{t}}\left[H_{t}^{T} R_{t}^{-1}\left(y_{t}-h\left(\mu_{t-1}\right)-H_{t}\left(\theta-\mu_{t-1}\right)\right]\right. \\
& =\mu_{t-1}+P_{t-1} H_{t}^{T} R_{t}^{-1}\left(y_{t}-h\left(\mu_{t-1}\right)+H_{t} \mu_{t-1}\right)-P_{t-1} H_{t}^{T} R_{t}^{-1} H_{t} \mu_{t} \\
& \Longleftrightarrow \\
\mu_{t} & =\left(\mathbf{I}+P_{t-1} H_{t}^{T} R_{t}^{-1} H_{t}\right)^{-1}\left(\mu_{t-1}+P_{t-1} H_{t}^{T} R_{t}^{-1}\left(y_{t}-h\left(\mu_{t-1}\right)+H_{t} \mu_{t-1}\right)\right) \\
& =\left(P_{t-1}^{-1}+H_{t}^{T} R_{t}^{-1} H_{t}\right)^{-1} P_{t-1}^{-1}\left(\mu_{t-1}+P_{t-1} H_{t}^{T} R_{t}^{-1}\left(y_{t}-h\left(\mu_{t-1}\right)+H_{t} \mu_{t-1}\right)\right) \\
& =\mu_{t-1}+\left(P_{t-1}^{-1}+H_{t}^{T} R_{t}^{-1} H_{t}\right)^{-1}\left(-H_{t}^{T} R_{t}^{-1} H_{t} \mu_{t-1}+H_{t}^{T} R_{t}^{-1}\left(y_{t}-h\left(\mu_{t-1}\right)+H_{t} \mu_{t-1}\right)\right) \\
& =\mu_{t-1}+P_{t} H_{t}^{T} R_{t}^{-1}\left(y_{t}-h\left(\mu_{t-1}\right)\right) \\
& =\mu_{t-1}-P_{t} \nabla_{\theta} \ell_{t}\left(\mu_{t-1}\right) \\
& =\mu_{t-1}-P_{t} \mathbf{E}_{q_{t-1}}\left[\nabla_{\theta} \tilde{\ell}_{t}(\theta)\right] \\
& =\mu_{t-1}+K_{t}\left(y_{t}-h\left(\mu_{t-1}\right)\right) .
\end{aligned}
$$

From (50) and (118), we deduce that R-VGA is equivalent to the explicit scheme.

From (50) and (119), we deduce that R-VGA is equivalent to the extended Kalman filter using the same formula (110) and (111) as in the linear case. The equivalence between the extended Kalman filter and the natural gradient is already known [Ollivier, 2018], we recall the main argument. In the update equation (50), the information matrix $P_{t}^{-1}$ is of growing size as long as we observe new data. If we pose $J_{t}=\frac{1}{t+1} P_{t}^{-1}$, we 
can reformulate the update (50) as a moving average:

$$
\begin{aligned}
J_{t} & =\frac{t}{t+1} J_{t-1}+\frac{1}{t+1} H_{t}^{T} R_{t}^{-1} H_{t} \\
& =\frac{t}{t+1} J_{t-1}+\left.\frac{1}{t+1} \mathbf{E}_{y}\left[\frac{\partial \log p(y \mid \theta)}{\partial \theta} \frac{\partial \log p(y \mid \theta)^{T}}{\partial \theta}\right]\right|_{\mu_{t-1}} \\
& =\frac{t}{t+1} J_{t-1}-\left.\frac{1}{t+1} \mathbf{E}_{y}\left[\frac{\partial^{2} \log p(y \mid \theta)}{\partial \theta^{2}}\right]\right|_{\mu_{t-1}} \\
& =\frac{t}{t+1} J_{t-1}+\left.\frac{1}{t+1} \mathbf{E}_{y}\left[\nabla_{\theta}^{2} \ell_{t}(\theta)\right]\right|_{\mu_{t-1}}
\end{aligned}
$$

which is the Fisher matrix update. The derivation from (120) to (121) is not obvious. Martens [2020] introduces it in the context of the generalized Gauss-Newton. To better understand where it comes from we rather use the proof proposed in Ollivier [2018]. Using the relation $\frac{\partial \log p(y \mid \theta)}{\partial \eta}=y-\bar{y}$ which holds for exponential families, we can write:

$$
\begin{aligned}
\frac{\partial \bar{y}}{\partial \theta} R_{t}^{-1} \frac{\partial \bar{y}^{T}}{\partial \theta} & =\frac{\partial \bar{y}}{\partial \theta} R_{t}^{-1} R_{t} R_{t}^{-1} \frac{\partial \bar{y}^{T}}{\partial \theta} \\
& =\frac{\partial \bar{y}}{\partial \theta} \frac{\partial \eta}{\partial \bar{y}} R_{t} \frac{\partial \eta^{T}}{\partial \bar{y}} \frac{\partial \bar{y}^{T}}{\partial \theta}=\frac{\partial \eta}{\partial \theta} R_{t} \frac{\partial \eta^{T}}{\partial \theta} \\
& =\frac{\partial \eta}{\partial \theta} \mathbf{E}_{y}\left[(y-\bar{y})(y-\bar{y})^{T}\right] \frac{\partial \eta^{T}}{\partial \theta} \\
& =\frac{\partial \eta}{\partial \theta} \mathbf{E}_{y}\left[\frac{\partial \log p(y \mid \theta)}{\partial \eta} \frac{\partial \log p(y \mid \theta)^{T}}{\partial \eta}\right] \frac{\partial \eta^{T}}{\partial \theta} \\
& =\mathbf{E}_{y}\left[\frac{\partial \log p(y \mid \theta)}{\partial \theta} \frac{\partial \log p(y \mid \theta)^{T}}{\partial \theta}\right] .
\end{aligned}
$$

\subsection{Output uncertainty assessment}

Given an unseen input, a prediction based on the estimated distribution $q_{t}$, may either be obtained through the maximum a posteriori (MAP) estimate of the parameter, that is, $P[y \mid x]=\sigma\left(x^{T} \theta^{*}\right)$, or it may be obtained using the entire (Bayesian) distribution, that is, $P[y \mid x]=\mathbf{E}[y \mid x]=\mathbf{E}_{q \sim \mathscr{N}\left(\mu^{*}, P^{*}\right)}\left[\sigma\left(x^{T} \theta\right)\right] \approx \sigma\left(k x^{T} \mu^{*}\right)$ with $k=\frac{\beta}{\sqrt{x^{T} P^{*} x+\beta^{2}}}$. Because of this, the latter is less confident than the former MAP based-approach. Indeed we have the following relation for the sigmoid:

$$
\left|\mathbf{E}_{q}[\sigma(x)]-0.5\right|<\left|\sigma\left(\mathbf{E}_{q}[x]\right)-0.5\right| \text { for } k<1 .
$$

This relation comes from the fact the sigmoid is convex for $x<0$ and concave for $x>0$. The prediction based on the Bayesian approach are shown in figure 11 where we have drawn the iso-probabilities of the outputs in function of the inputs. On this separable data-set, the Laplace give low probabilities prediction for the unseen inputs whereas the QKF tends to predict with high probabilities. The R-VGA gives prediction probabilities between both of them. 

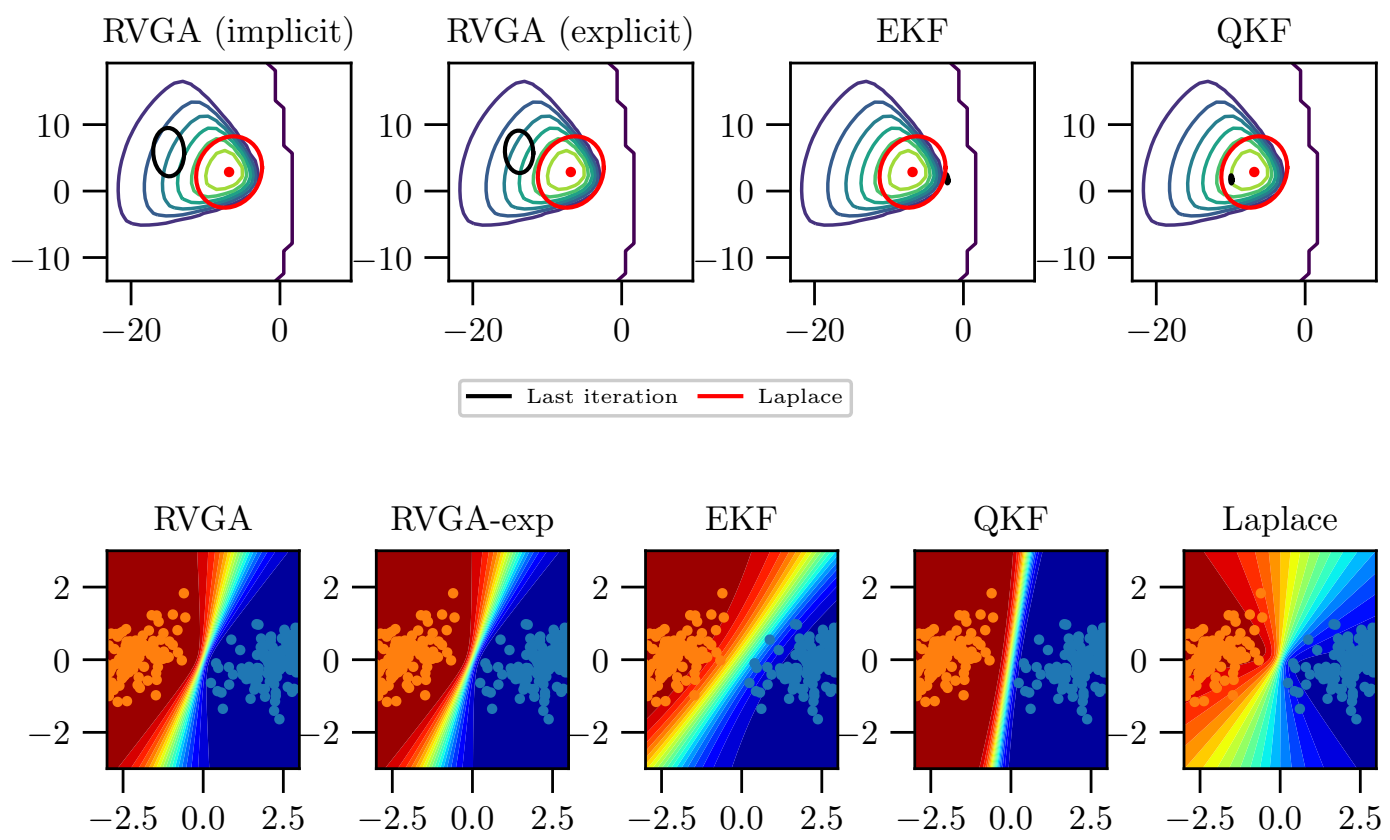

Figure 11: Iso-probabilities of the outputs in function of the inputs for the R-VGA implicit and explicit schemes (left), the EKF, QKF (middle) and the Laplace (right). We have considered $\sigma_{0}=10$ and $\mu_{0}=0$ with $N=300$ and $s=5$.

\subsection{Details on the fixed point method}

The roots of $F$ are the fixed point of the function $\tilde{F}$ defined by :

$$
\begin{aligned}
\tilde{F}_{\alpha_{0}, v_{0}, y}: \mathbb{R}^{2} & \rightarrow \mathbb{R}^{2} \\
(\alpha, v) & \rightarrow \tilde{F}(\alpha, v)=(\tilde{f}, \tilde{g})
\end{aligned}
$$

where:

$$
\begin{aligned}
& \tilde{f}(\alpha, v)=-v_{0} \sigma(\alpha k(v))+\alpha_{0}+v_{0} y \\
& \tilde{g}(\alpha, v)=\frac{v_{0}}{1+v_{0} k(v) \sigma^{\prime}(\alpha k(v))} .
\end{aligned}
$$

Function $F$ is displayed in Figure 12. We found that $\tilde{F}$ is not contractive, so that fixed point iterations shall oscillate and do not converge, see Figure 13. However we can further restrict the admissible domain $\left[\alpha_{\min }, \alpha_{\max }\right] \times\left[v_{\min }, v_{\max }\right]$ in which the searched point lies as follows.

Coarse bounds are given by:

$$
\begin{aligned}
& \alpha_{\text {min }}=\alpha_{0}+v_{0}(y-1) \leq \alpha \leq \alpha_{0}+v_{0} y=\alpha_{\text {max }} \\
& v_{\text {min }}=v_{0}\left(1-\frac{v_{0}}{4+v_{0}}\right) \leq v \leq v_{0}=v_{\text {max }} .
\end{aligned}
$$



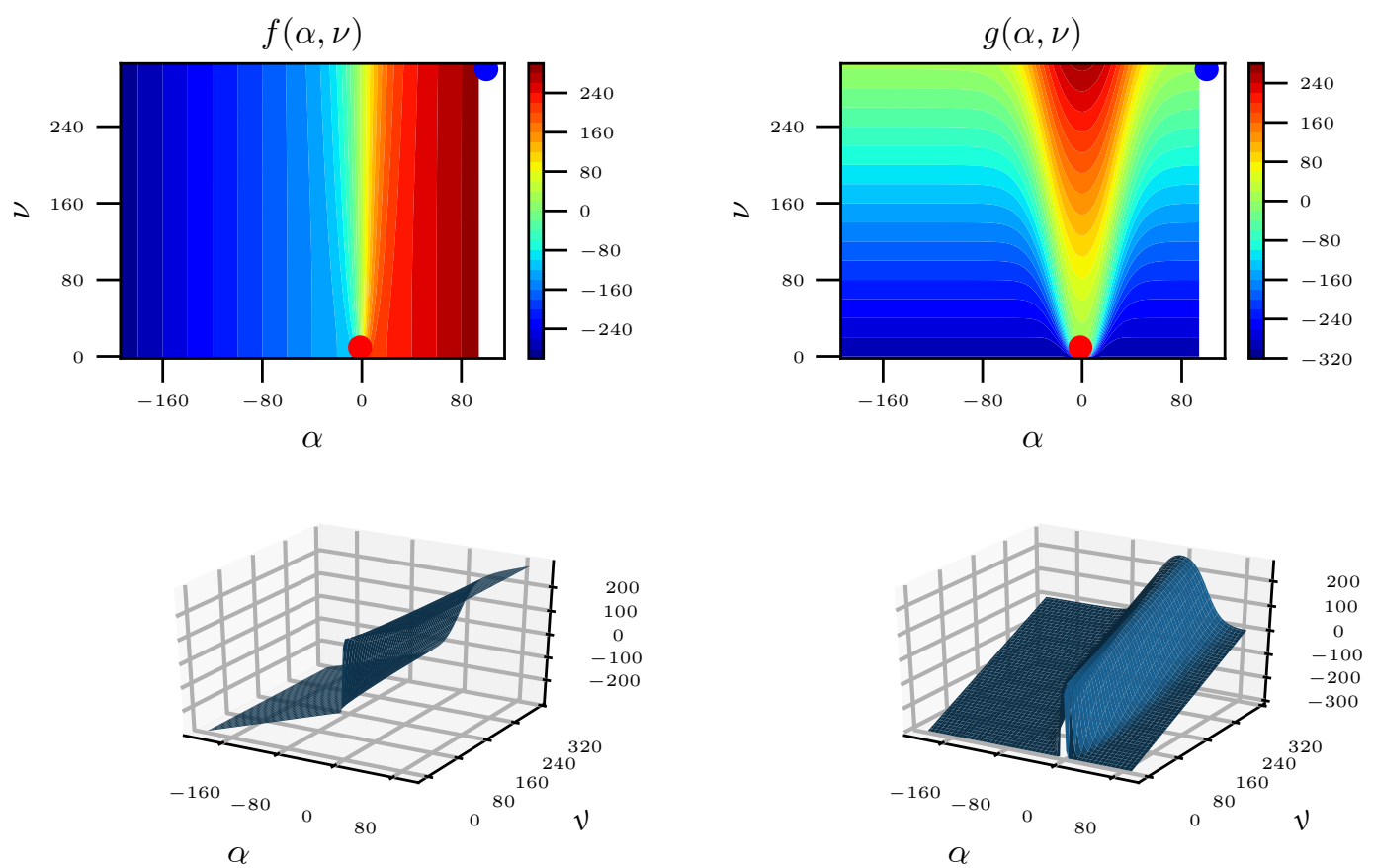

Figure 12: Values of the function $F_{\alpha_{0}, v_{0}, y}$ for $\alpha_{0}=100, v_{0}=300$ and $y=0$. In the upper row, we show the initial value $\left(\alpha_{0}, v_{0}\right)$ which is located on the bound of the domain (blue dot) and the optimal value (red dot).

Using the fact that $0 \leq k\left(v_{\max }\right) \leq k(v) \leq k\left(v_{\min }\right)$, the first inequality (127) gives:

$$
\begin{array}{cc} 
& a_{1} \leq \alpha k(v) \leq a_{2} \\
& \\
\text { where: } & \\
\alpha_{\text {min }} \geq 0 \Rightarrow a_{1}=\alpha_{\text {min }} k\left(v_{\text {max }}\right), & \alpha_{\text {min }}<0 \Rightarrow a_{1}=\alpha_{\text {min }} k\left(v_{\text {min }}\right) \\
\alpha_{\text {max }} \geq 0 \Rightarrow a_{2}=\alpha_{\text {max }} k\left(v_{\text {min }}\right), & \alpha_{\text {max }}<0 \Rightarrow a_{2}=\alpha_{\text {max }} k\left(v_{\text {max }}\right)
\end{array}
$$

And we find the following new bound for $\alpha$ :

$$
\alpha_{0}+v_{0} y-v_{0} \sigma\left(a_{2}\right) \leq \alpha \leq \alpha_{0}+v_{0} y-v_{0} \sigma\left(a_{1}\right) .
$$

For the second inequality (128) we use (129) to bound :

$$
b_{1} \leq \sigma(\alpha k(v))(1-\sigma(\alpha k(v)))=\sigma^{\prime}(\alpha k(v)) \leq b_{2},
$$

where $b_{1}$ and $b_{2}$ depends on the sign of $a_{1} a_{2}$ :

$$
\begin{aligned}
& a_{1} a_{2}>0 \Rightarrow b_{1}=\min \left(\sigma^{\prime}\left(a_{1}\right), \sigma^{\prime}\left(a_{2}\right)\right) \text { and } b_{2}=\max \left(\sigma^{\prime}\left(a_{1}\right), \sigma^{\prime}\left(a_{2}\right)\right) \\
& a_{1} a_{2} \leq 0 \Rightarrow b_{1}=\min \left(\sigma^{\prime}\left(a_{1}\right), \sigma^{\prime}\left(a_{2}\right)\right) \text { and } b_{2}=1 / 4 .
\end{aligned}
$$

And we find the following new bound for $v$ :

$$
\frac{v_{0}}{1+v_{0} k\left(v_{\min }\right) b_{2}} \leq v \leq \frac{v_{0}}{1+v_{0} k\left(v_{\max }\right) b_{1}} .
$$

This scheme can be iterated to restrict the search domain.

For moderate values of $v_{0}$, that is moderately uncertain prior, the domain shrinks fast. But for highly uncertain priors it does not, as shown in Figure 13, hence the need to resort to a 2D optimization algorithm. 

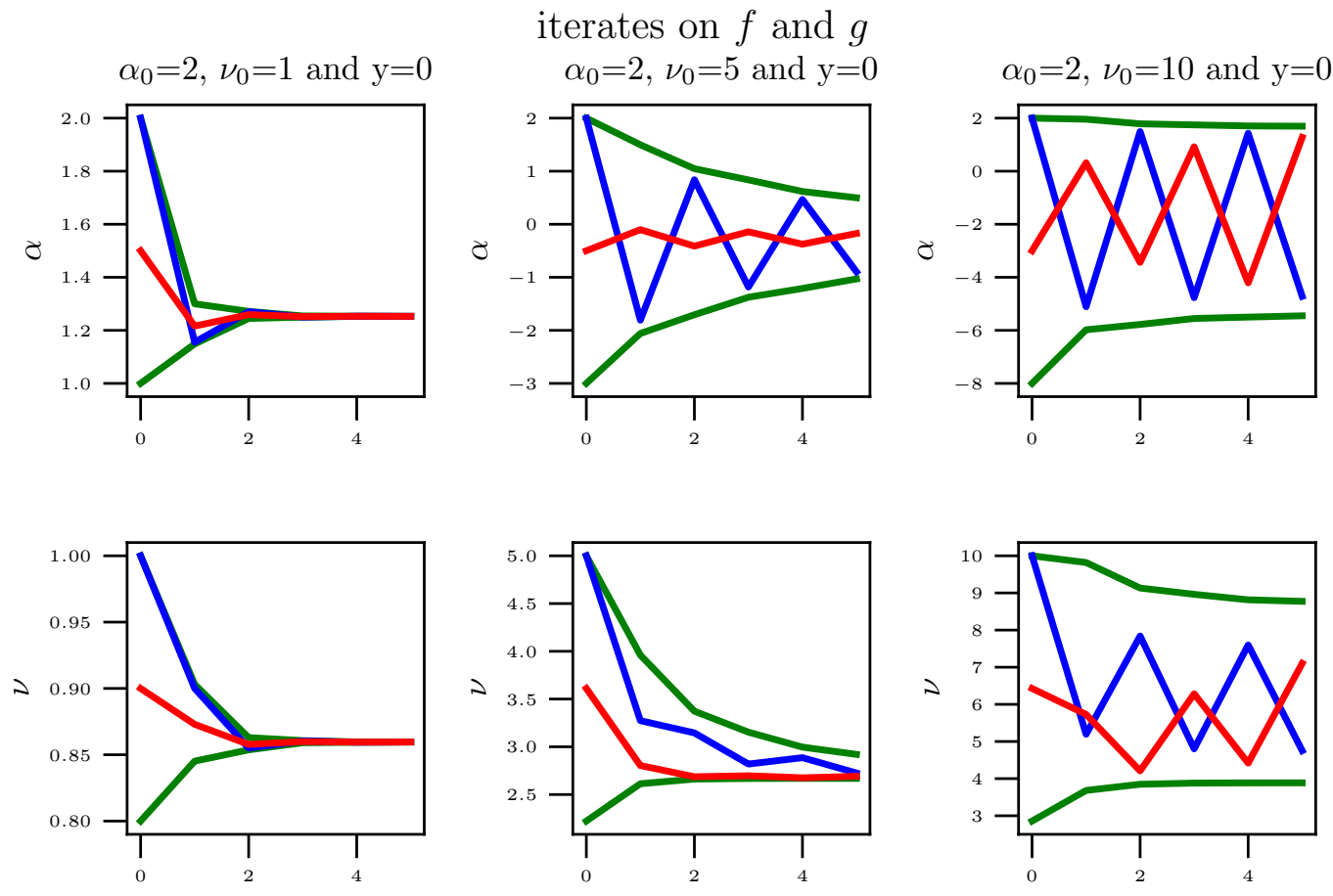

number of iterations

number of iterations

number of iterations

Figure 13: Bounds for the roots $\alpha, v$ of $F$ through the proposed iterative scheme (in green) for $v_{0}=1,5$ and 10. Iteration on $F$ for different initial conditions are shown in red and blue.

\subsection{Influence of separability of the dataset}

We plot here results that reflect the sensitivity to the separability factor $s$ with $s=2$ (Figure 14), $s=5$ (Figure $15)$ and $s=10$ (Figure 16). The evolution of the ellipsoids over the iterations are also displayed. We see RVG-A consistently yields good performance. 

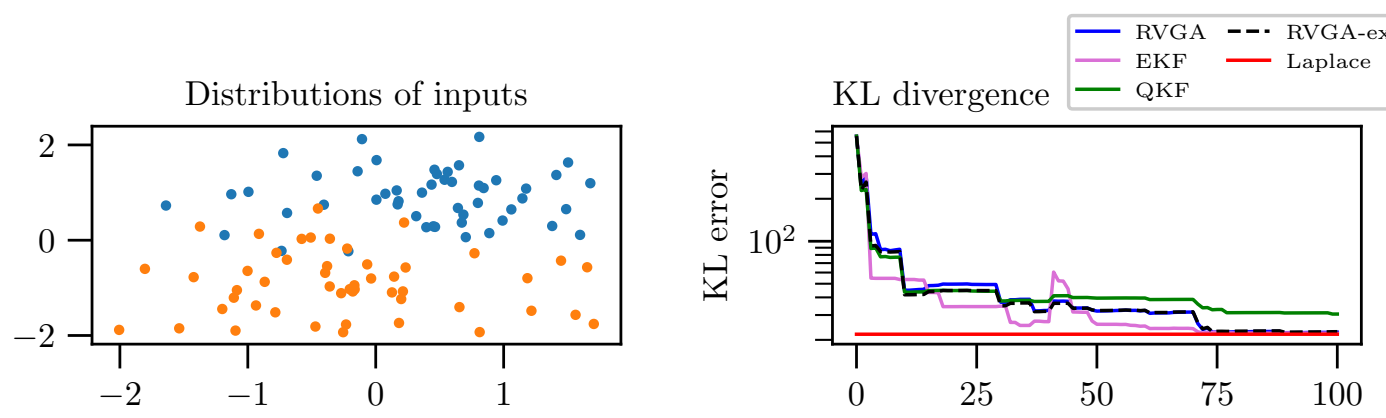

$\sigma_{0}=10,\left\|\mu_{0}\right\|=0.0, \mathrm{~N}=100, \mathrm{~d}=2, \mathrm{~s}=2.0, \mathrm{c}=1$

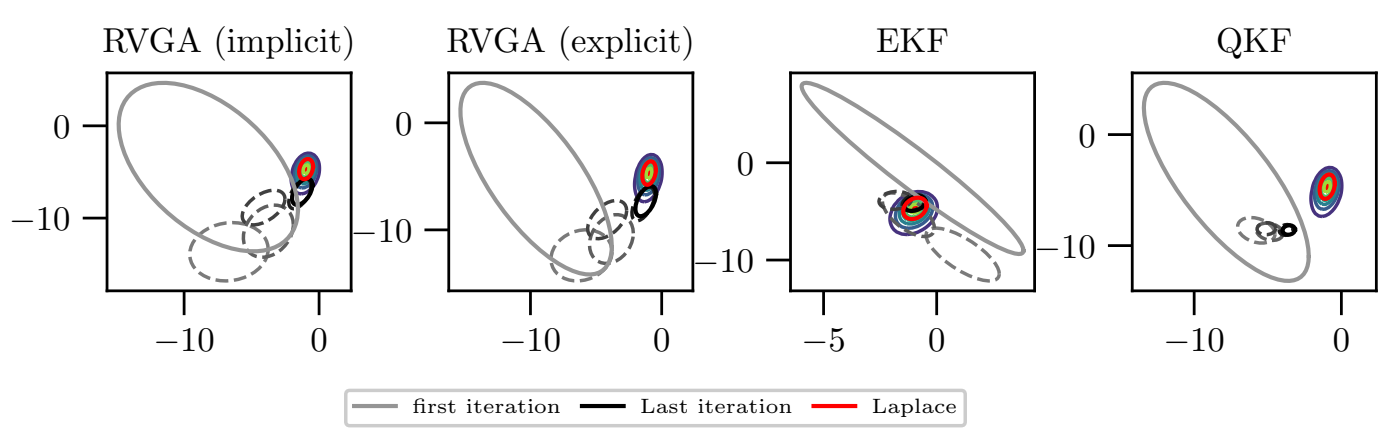

Figure 14: $s=2$. Upper row: 2D synthetic data-set (left) and KL divergence. Lower row: Confidence ellipsoids for the R-VGA implicit and explicit versions (left) and the EKF and QKF (right) at different times (final $t=N$ in black). The Batch Laplace covariance is shown in red and is considered accurate.
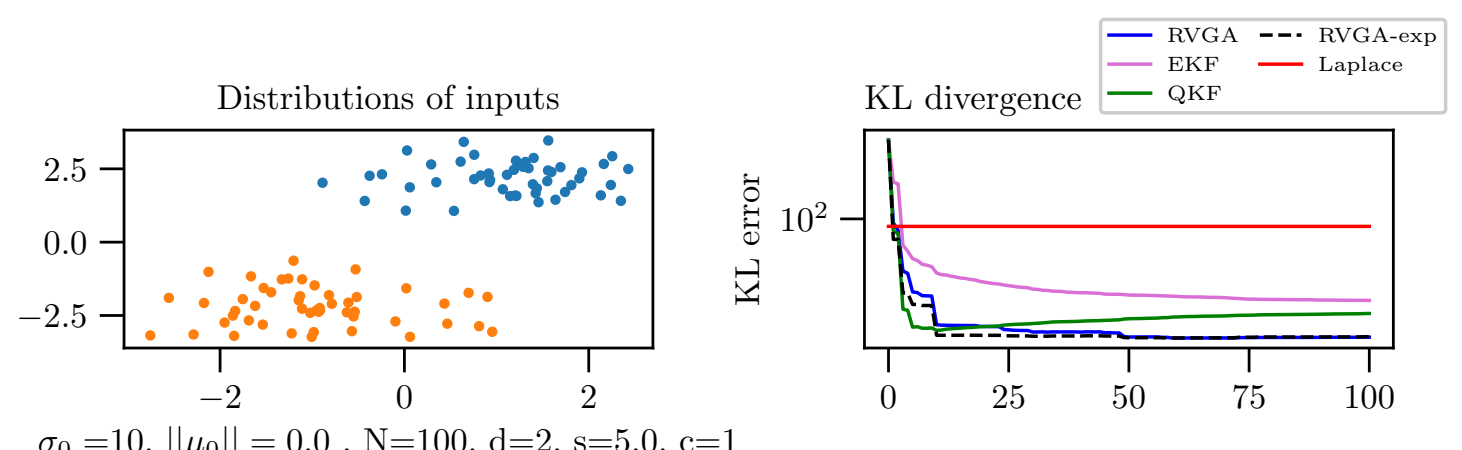

$\sigma_{0}=10,\left\|\mu_{0}\right\|=0.0, \mathrm{~N}=100, \mathrm{~d}=2, \mathrm{~s}=5.0, \mathrm{c}=1$
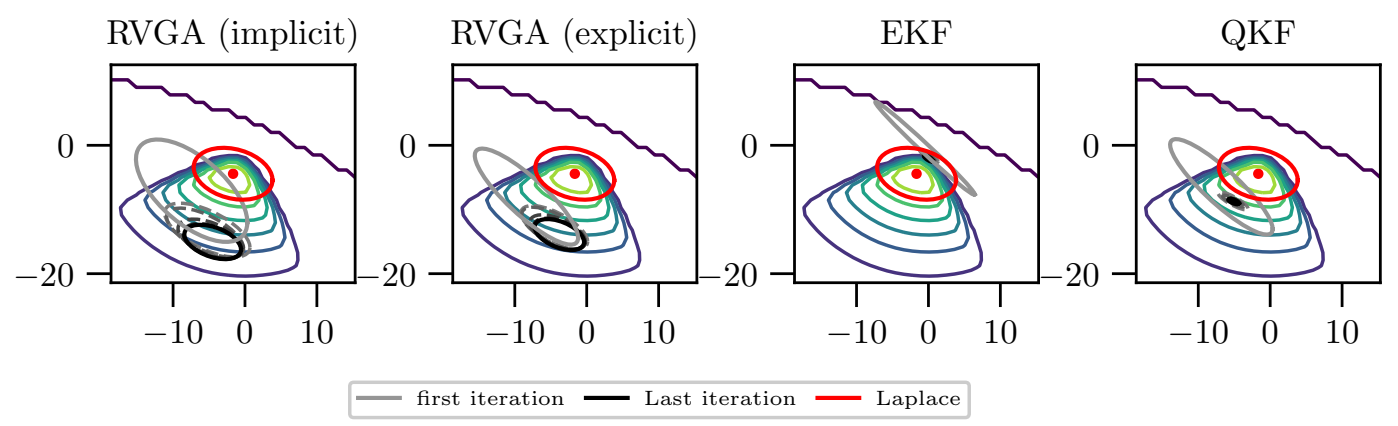

Figure 15: Same as Figure 14 in the case where $s=5$. 


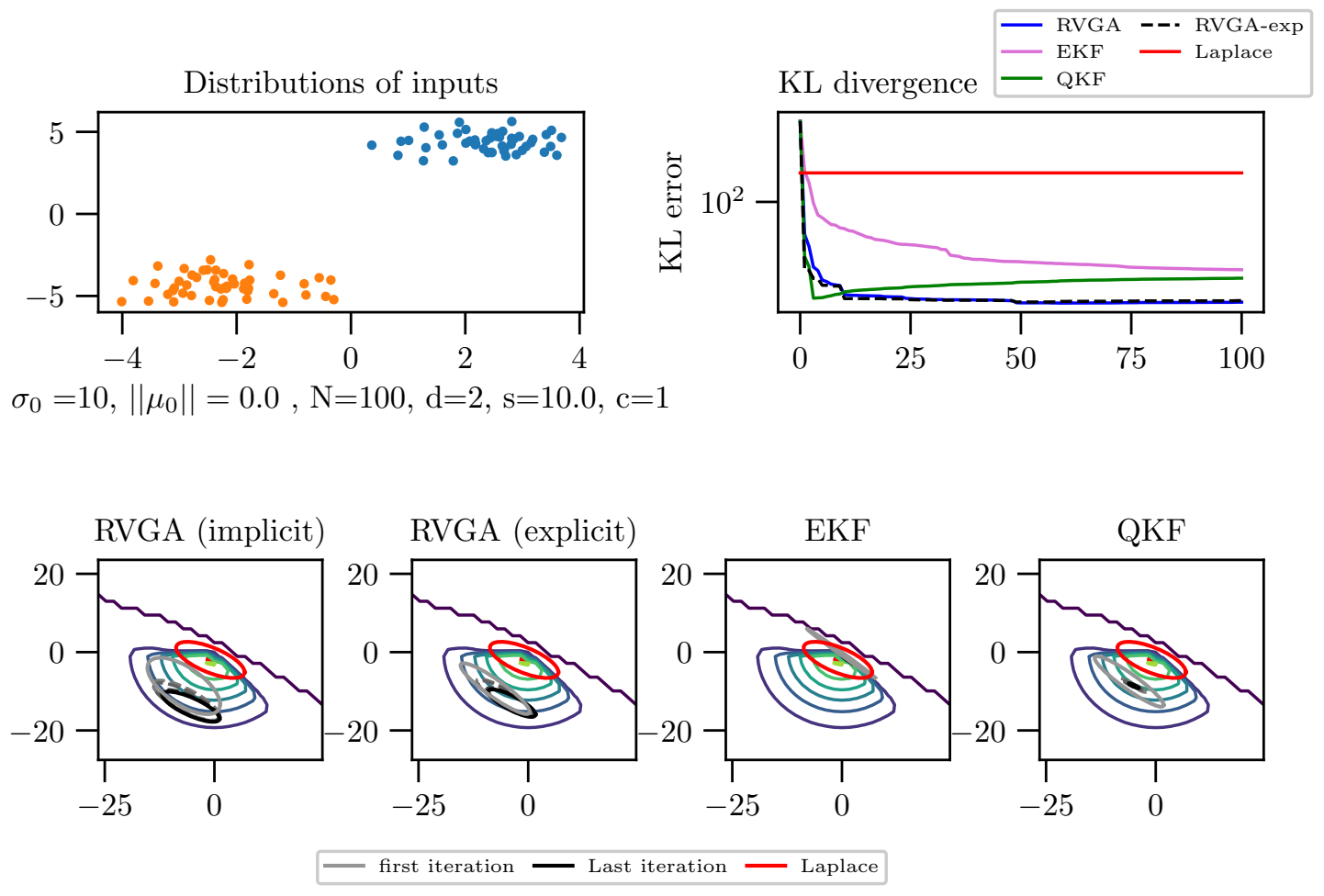

Figure 16: Same as Figure 14 in the case where $s=10$. 